\title{
A FLUID CLUSTER POISSON INPUT PROCESS CAN LOOK LIKE A FRACTIONAL BROWNIAN MOTION EVEN IN THE SLOW GROWTH AGGREGATION REGIME
}

\author{
VICKY FASEN, ${ }^{*}$ Technische Universität München \\ GENNADY SAMORODNITSKY,** Cornell University
}

\begin{abstract}
We show that, contrary to common wisdom, the cumulative input process in a fluid queue with cluster Poisson arrivals can converge, in the slow growth regime, to a fractional Brownian motion, and not to a Lévy stable motion. This emphasizes the lack of robustness of Lévy stable motions as 'birds-eye' descriptions of the traffic in communication networks.
\end{abstract}

Keywords: Cluster Poisson process; fluid queue; fractional Brownian motion; input model; slow growth regime; scaling limit; workload process

2000 Mathematics Subject Classification: Primary 90B22

Secondary 60F17

\section{Introduction}

This paper concerns the asymptotic behavior of certain fluid random streams of the type that have often been taken as natural models of input for fluid queues and queueing networks. We are specifically interested in the effect of heavy tails on such asymptotic behavior. We do not consider the actual queues in this paper, in the sense that we investigate only the potential input process to a queue and not what happens once the service starts. However, our task, which lies in understanding how the input process deviates from its completely regular and linear average behavior, will facilitate the understanding of how an actual queue with such input behaves. Indeed, it is precisely the deviations from the average behavior that build the queue.

The motivation for our interest in deviations of input processes from their average (as well as the motivation behind many other papers on this subject) lies in the fact that networks with heavy-tailed inputs are difficult to analyze, since they are not well suited to Brownian or Poisson approximations. Nonetheless, it is believed that heavy tails cause unusual (and often negative) effects. For example, it is believed that infinite variance in the distribution of the file sizes or bandwidth requests in communication networks causes long-range dependence and self-similar structure in the network (see, e.g. Park and Willinger (2000)).

Since queues with heavy-tailed input are difficult to analyze directly, the hope has been to obtain insight into their behavior by approximating the input by something standard, specifically by the average, linear, stream plus a certain deviation from that average. In the influential paper

Received 27 May 2008; revision received 13 February 2009.

* Postal address: Center for Mathematical Sciences, Technische Universität München, D-85747 Garching, Germany. Email address: fasen@ma.tum.de

** Postal address: School of Operations Research and Information Engineering, Cornell University, 206 Rhodes Hall, Ithaca, NY 14853, USA. Email address: gennady@ orie.cornell.edu 
of Mikosch et al. (2002) they showed that, for the so-called ON/OFF model and the infinitesource Poisson model, the properly compensated and normalized cumulative input in a fluid queue looks like a fractional Brownian motion in the fast growth regime and like a Lévy stable motion in the slow growth regime. This result was later extended to networks of fluid queues in D'Auria and Samorodnitsky (2005). A random field version of such results is given in Kaj et al. (2007). The terms fast growth regime and slow growth regime are important. They refer to the relative magnitude of the time scale at which the input process is considered and the number of independent streams the input consists of. We will return to this important point shortly. Boundary regimes have been discovered as well; see, e.g. Gaigalas and Kaj (2003).

These previous results appear to indicate that the deviations from the average in a heavytailed input process could look, in the limit, as either a fractional Brownian motion or a Lévy stable motion. These are two very different stochastic processes: one has light tails but strongly dependent increments, while the other has independent increments but heavy tails. We expect very different performance in a queue with such different inputs. What was needed, therefore, was a study of the robustness of these two possible limits under departures from the very specific model assumptions of Mikosch et al. (2002). Such a study was undertaken in Mikosch and Samorodnitsky (2007) in a general setup, described below. Consider a stationary marked point process

$$
\left(\left(\mathcal{T}_{m}, Z_{m}\right)\right)_{m \in \mathbb{Z}},
$$

where a possible interpretation in the language of communication systems views $\cdots<\mathcal{T}_{-1}<$ $\mathcal{T}_{0}<0<\mathcal{T}_{1}<\cdots$ as the arrival times of data packets at a server and $Z_{m}$ as the file size of the data packet transmitted at $\mathcal{T}_{m}$. Each arrival corresponds to a 'source', and it transmits its data at a unit rate. Thus, $Z_{m}$ also denotes the transmission period. The number of active sources at time $t$ is given by the process

$$
U(t)=\sum_{m \in \mathbb{Z}} \mathbf{1}_{\left\{\mathcal{T}_{m} \leq t<\mathcal{T}_{m}+Z_{m}\right\}} \quad \text { for } t \geq 0,
$$

and the amount of data transmitted to the server in the interval $[0, t]$ is given by the input process

$$
A(t)=\int_{0}^{t} U(y) \mathrm{d} y=\sum_{m \in \mathbb{Z}}\left[Z_{m} \wedge\left(t-\mathcal{T}_{m}\right)-Z_{m} \wedge\left(-\mathcal{T}_{m}\right)\right], \quad t \geq 0,
$$

which has continuous sample paths and, thus, reflects the fluid queue. Under the assumption that the marks $\left(Z_{m}\right)$ have, under the Palm measure, a finite mean, $A(t)$ has a finite mean with $\mathrm{E}(A(t))=\mu t$, where $\mu>0$ is the expected amount of data arriving at the server in $[0,1]$.

Let $\left(A_{i}\right)_{i \in \mathbb{N}}$ be independent and identically distributed (i.i.d.) copies of the process $A$. We view each $\left(A_{i}\right)$ as the input process of data generated by the $i$ th 'user', with different users having nothing to do with each other; hence, the independence assumption. With $n$ such independent input processes and at a time scale $M$, the deviation of the cumulative input process from its mean is the stochastic process

$$
D_{n, M}(t)=\sum_{i=1}^{n}\left(A_{i}(t M)-\mu t M\right) \quad \text { for } t \geq 0 .
$$

We are interested in the limits of the sequence of processes $\left(D_{n, M}\right)$ as $n$ and $M$ grow to $\infty$. It is here where the idea of 'fast growth' and 'slow growth' appears. 
The terms fast growth regime and slow growth regime were introduced in Mikosch et al. (2002) for the ON/OFF and infinite-source Poisson models, and they described the relative rates at which $n$ and $M$ in (1.3) grow to $\infty$. Intuitively, the fast growth regime refers to the situation where the number of the input processes, $n$, is relatively large in comparison to the time scale $M$, while the slow growth regime refers to the opposite situation. In fact, Mikosch et al. (2002) used a very specific boundary: $n(M) \uparrow \infty$ as $M \uparrow \infty$ such that the fast growth regime meant that $n \gg n(M)$, while the slow growth regime meant that $n \ll n(M)$. There are substantial reasons to separate the regimes. Indeed, if the number of sources, $n$, is very large then the process $\left(D_{n, M}(t), t \geq 0\right)$ in (1.3) is the sum of a very large number of i.i.d. terms that change relatively slowly. If the number of active sources in (1.1) has a finite variance (as is the case in most systems considered in the literature), the same would be the case for the input process in (1.2). Then we would expect a Gaussian limit for the deviation from the mean of the cumulative input process as in a classical central limit theorem. On the other hand, if the time scale $M$ is very large then the main phenomenon in (1.3) is, actually, the deviations of the individual input processes from their means, $A_{i}(t)-\mu t$ for large $t$. A priori, there is no reason to expect these latter deviations to be Gaussian-looking, unless very specific assumptions are imposed on the generic input process $(A(t), t \geq 0)$. These assumptions are not of the kind usually imposed in the literature on the input to communication systems. Correspondingly, Mikosch et al. (2002) discovered that, in the ON/OFF and infinite-source Poisson models, the deviation from the mean of the cumulative input process has a stable limit in the slow growth regime.

We take a related, but somewhat more general, point of view on the notions of the fast growth regime and the slow growth regime, which has already been used in Mikosch and Samorodnitsky (2007). Specifically, if there is a function $n(M) \uparrow \infty$ as $M \uparrow \infty$ such that a limit theorem holds when $n \gg n(M)$, we say that this limit theorem holds in the fast growth regime because the main effect in (1.3) is the averaging over the many independent input streams. In fact, this regime typically allows an iterated limiting procedure: first let $n \rightarrow \infty$ and then let $M \rightarrow \infty$.

Similarly, if there is a function $n(M) \uparrow \infty$ as $M \uparrow \infty$ such that a limit theorem holds when $n \ll n(M)$, we say that this limit theorem holds in the slow growth regime because the main effect in (1.3) is the deviations of the individual input processes from their means, and the limit will typically hold if we first let $M \rightarrow \infty$ and then let $n \rightarrow \infty$.

It has become a part of the folklore that in the former scenario a fractional Gaussian limit is likely to arise, while in the latter scenario a Lévy stable limit can be expected. What Mikosch and Samorodnitsky (2007) discovered was that the fractional Brownian limits of Mikosch et al. (2002) in the fast growth regime were very robust, and held under very general assumptions on the underlying stationary marked point process. On the other hand, the Lévy stable limits turned out to be nonrobust, and very special conditions were needed to ensure such limits. One of the conclusions of Mikosch and Samorodnitsky (2007) was that, in certain circumstances of a very irregular arrival process, a fractional Brownian limit was possible even in the slow growth regime. They provided a somewhat artificial example of such a situation, and conjectured that the same was true in the important case of a cluster Poisson arrival process. It is the purpose of this paper to consider that case and establish the fractional Brownian limit. Once this is accomplished, we understand that the appearance of a fractional Brownian limit in the slow growth regime is not exotic but, in fact, can be possible under very natural and common assumptions. This emphasizes how robust the fractional Brownian motion limiting behavior is. In a related work, a reflected version of the fractional Brownian limit was established in Delgado (2007) for the workload in fluid queueing networks in a heavy-traffic regime. 
We would like to mention at this point that, for certain input point processes, changing the number of independent input streams, $n$, as above is equivalent to changing the intensity $\lambda_{0}$ of an underlying Poisson process. This is true for the M/G/ $\infty$ model of Mikosch et al. (2002), and it is also true for the model considered in the present paper. For such point processes, it is possible (and natural) to distinguish between different situations according to the relative rates at which the Poisson intensity and the time scale grow to $\infty$. Accordingly, if there exists a function $\lambda(M) \uparrow \infty$ such that a limit theorem holds if $\lambda_{0} \gg \lambda(M)$, we say that the limit holds in the fast growth regime, and if a limit theorem holds under the assumption $\lambda_{0} \ll \lambda(M)$, we say that the limit holds in the slow growth regime. In this paper we will use the fast and slow growth regimes terminology introduced above that compares the rates of growth of the number $m$ and the time scale $M$ because this is the terminology in which a key result of Mikosch and Samorodnitsky (2007), which we use in this paper, is stated (see Theorem 3.2, below).

This paper is arranged as follows. The arrival cluster Poisson model we are working with is formally described in Section 2. The main result of the paper is stated and discussed in Section 3. The arguments required to prove the main result uses a number of renewal theoretical and extreme value results, some of which may be of independent interest. These appear in Section 4. In Section 5 we present the proof of the main theorem. Finally, Section 6 contains additional lemmas and other technical results needed for the proof of the main theorem.

\section{The cluster Poisson model}

We assume that the data file sizes $\left(Z_{m}\right)_{m \in \mathbb{Z}}$ form an i.i.d. sequence independent of the arrival process $\left(\mathcal{T}_{n}\right)_{n \in \mathbb{Z}}$. Let the number of sources arriving at the server in the interval $(s, t]$ be described by

$$
N(s, t]=\sum_{m \in \mathbb{Z}} \mathbf{1}_{\left\{s<\mathcal{T}_{m} \leq t\right\}} \quad \text { for } s<t .
$$

Furthermore, we assume that this arrival point process is a cluster Poisson process. Specifically,

(i) initial cluster points, denoted by $\cdots<\Gamma_{-1}<0<\Gamma_{1}<\Gamma_{2}<\cdots$, form a homogeneous Poisson process $\tilde{N}$ with rate $\lambda_{0}$;

(ii) at each initial cluster center $\Gamma_{m}$ an independent copy of a randomly stopped renewal point process $N_{c}$ starts.

A generic point process $N_{c}$ has the form

$$
N_{c}[0, t]=N_{0}[0, t] \wedge(K+1),
$$

where $N_{0}$ is a renewal point process with arrival times $0=T_{0}<T_{1}<\cdots$ and $K$ is a nonnegative integer-valued random variable independent of $N_{0}$. The interarrival times $X_{k}=$ $T_{k}-T_{k-1}$ for $k \geq 1$ are i.i.d. random variables, with a common distribution $F$, and the cluster size $K$ has distribution $F_{K}$. The cluster with the initial point $\Gamma_{m}$ has the points $\Gamma_{m, k}=\Gamma_{m}+T_{k, m}$ for $k=0, \ldots, K_{m}$, where $\left(T_{k, m}\right)_{k \in \mathbb{N}_{0}}$ are independent copies of $\left(T_{k}\right)_{k \in \mathbb{N}_{0}}$, independent of $\left(\Gamma_{m}\right)_{m \in \mathbb{Z}}$.

The within-cluster interarrival times and the cluster sizes are assumed to satisfy the following assumption.

Assumption 2.1. (a) The interarrival distribution function satisfies

$$
\bar{F} \in \mathcal{R}_{-1 / \beta} \quad \text { with } \beta>1 \text {. }
$$


(b) The cluster size distribution function satisfies

$$
\bar{F}_{K} \in \mathcal{R}_{-\alpha} \text { with } 1<\alpha<\min (2, \beta) .
$$

(c) The marks $\left(Z_{m}\right)$ form a sequence of i.i.d. random variables, independent of the underlying point process. Furthermore, we will assume that $\mathrm{E}\left(\left|Z_{m}\right|^{2}\right)<\infty$.

Note that Assumption 2.1(a) ensures that the within-cluster interarrival times have infinite mean; it also makes the arrival process sufficiently irregular for our result. Assumption 2.1(b) ensures that the data files transmitted within each cluster have infinite variance. Note that the intensity of $N$ is

$$
\lambda=\lambda_{0}(1+\mathrm{E}(K)) .
$$

For our main result, Theorem 3.1, below, we will introduce the following additional assumption on the interarrival distribution function $F$.

Assumption 2.2. Assume that either

1. $\beta<2$ and

$$
\limsup _{x \rightarrow \infty} x \frac{\bar{F}(x)-\bar{F}(x+1)}{\bar{F}(x)}<\infty
$$

or

2. $F$ is arithmetic with step size $\Delta>0$ and

$$
\limsup _{n \geq 0} n \frac{F(\{n \Delta\})}{\bar{F}(n \Delta)}<\infty .
$$

Remark 2.1. We need Assumption 2.2 to obtain a local renewal theorem; see Lemma 4.1, below, or Theorem 3 of Doney (1997). In fact, if the local renewal theorem is known to hold (if only in the form of an upper bound) then Assumption 2.2 is unnecessary. We conjecture that the local renewal theorem holds under (2.1) for any $\beta>1$, regardless of whether or not $F$ is arithmetic.

We denote by

$$
h(u)=\bar{F}^{\leftarrow}\left(\frac{1}{u}\right)=u^{\beta} l(u) \quad \text { for } u>1
$$

the generalized tail inverse function of the within-cluster interarrival time distribution (see Resnick (2006, Section 2.1.2)). Here, $l$ is a slowly varying function. One implication of Assumption 2.1(a) is the weak convergence

$$
\left(\frac{T_{\lfloor n t\rfloor}}{h(n)}\right)_{t \geq 0} \stackrel{\mathrm{w}}{\rightarrow}\left(S_{1 / \beta}(t)\right)_{t \geq 0}
$$

in $\mathscr{D}[0, \infty)$ as $n \rightarrow \infty$; see Kallenberg (2002, Theorem 16.14). Here $\left(S_{1 / \beta}(t)\right)_{t \geq 0}$ is a $1 / \beta$-stable subordinator. We will use the notation

$$
I(u)=\inf \left\{t \geq 0: S_{1 / \beta}(t)>u\right\} \quad \text { for } u>0
$$

for its inverse process. Then the weak convergence

$$
\left(\bar{F}(r) N_{0}(0, r u]\right)_{u \geq 0} \stackrel{\mathrm{w}}{\rightarrow}(I(u))_{u \geq 0}
$$


in $\mathscr{D}[0, \infty)$ as $r \rightarrow \infty$ holds. In particular, the process $(I(u))_{u \geq 0}$ is self-similar of index $1 / \beta$; cf. Meerschaert and Scheffler (2004).

We will continue using the notation $\stackrel{\mathrm{W}}{\rightarrow}$ ' for weak convergence, ' $\stackrel{\mathrm{P}}{\rightarrow}$ ' for convergence in probability, ' $\stackrel{v}{\rightarrow}$ ' for vague convergence, and $\stackrel{\text { FDD }}{\longrightarrow}$ ' for weak convergence of the finitedimensional distributions. For $x \in \mathbb{R}$, we write $x_{+}=\max (0, x)$. For two random variables $X$ and $Y$, the symbol $X \stackrel{\mathrm{D}}{=} Y$ means that $X$ has the same distribution as $Y$.

We will also adopt the following convention. We will use the notation $\alpha_{1}, \alpha_{2}, \beta_{1}$, and $\beta_{2}$ for positive numbers satisfying $\alpha_{1}<\alpha<\alpha_{2}$ and $\beta_{1}<\beta<\beta_{2}$, in the sense that the statements in the text where this notation appears hold for any choice of numbers satisfying the above conditions with, perhaps, different multiplicative constants.

\section{The main result}

Below is the main result of this paper. It describes a slow growth regime under which the properly normalized deviations from the mean process (1.3) converge to a fractional Brownian motion. For a positive sequence $M_{n} \uparrow \infty$ serving as the time scale for a system with $n$ input processes, we define

$$
b_{n}=\sqrt{n M_{n} \bar{F}\left(M_{n}\right)^{-2} \mathrm{P}\left(K>\bar{F}\left(M_{n}\right)^{-1}\right)} \text { for } n \geq 1 .
$$

The sequence $\left(b_{n}\right)_{n \in \mathbb{N}}$ turns out to be the right normalization for process (1.3).

Theorem 3.1. Let the Poisson cluster model satisfy Assumptions 2.1 and 2.2. Furthermore, let $M_{n}$ be a sequence of positive constants such that $M_{n} \uparrow \infty$ and such that $b_{n}$ in (3.1) satisfies

$$
\lim _{n \rightarrow \infty} n b_{n}^{-(\alpha-1) / \beta+\rho}=0 \text { for some } \rho>0 .
$$

Then the cumulative input process $S_{n}(t)=b_{n}^{-1} D_{n, M_{n}}(t), n \geq 1, t \geq 0$, satisfies

$$
\left(S_{n}(t)\right)_{t \geq 0} \stackrel{\mathrm{FDD}}{\longrightarrow}\left(\mathrm{E}(Z) B_{H}(t)\right)_{t \geq 0} \text { as } n \rightarrow \infty,
$$

and the limiting process $B_{H}$ is a fractional Brownian motion with

$$
H=\frac{2+\beta-\alpha}{2 \beta} \in(0.5,1)
$$

and

$$
\begin{aligned}
\operatorname{var}\left(B_{H}(1)\right)= & \frac{2 \lambda_{0}}{2+\beta-\alpha} \int_{0}^{\infty} y^{-(2+\beta-\alpha) / \beta} \mathrm{P}\left(S_{1 / \beta}(1) \leq y\right) \mathrm{d} y \\
+ & \lambda_{0} \int_{0}^{\infty} \mathrm{E}\left(\frac{2}{2-\alpha} I(w+1)^{2-\alpha}+\frac{2}{\alpha-1} I(w) I(w+1)^{1-\alpha}\right. \\
& \left.-\frac{2}{(2-\alpha)(\alpha-1)} I(w)^{2-\alpha}\right) \mathrm{d} w
\end{aligned}
$$

where $\left(S_{1 / \beta}(t)\right)_{t \geq 0}$ and $(I(w))_{w \geq 0}$ are as in (2.3) and (2.4), respectively. 
Remark 3.1. Note that, for any $\varepsilon>0$, there exist $C>1$ such that

$$
C^{-1} n^{1 / 2} M_{n}^{H-\varepsilon} \leq b_{n} \leq C n^{1 / 2} M_{n}^{H+\varepsilon} \text { for } n \geq 1,
$$

with $H$ given by (3.3). Hence, a necessary and sufficient condition for (3.2) is that, for some $\varepsilon>0$,

$$
M_{n} \gg n^{(2 \beta-\alpha+1) / 2 H(\alpha-1)+\varepsilon} .
$$

This identifies (3.2) as a slow growth condition and explains the appearance of this term in the title of the paper.

We will prove Theorem 3.1 by showing that the assumptions of Theorem 5.2 of Mikosch and Samorodnitsky (2007) are satisfied. For convenience, we state that theorem below, in a form simplified for the situation, where the marks are independent of the arrival process.

Theorem 3.2. (Mikosch and Samorodnitsky (2007, Theorem 5.2).) Consider a marked stationary point process, where the marks $\left(Z_{m}\right)$ are independent of the arrival process $N$ (whose intensity is $\lambda$ ) and have a finite first moment. Let $M_{n}$ be a sequence of positive constants with $M_{n} \uparrow \infty$. Suppose that there exists a sequence $b_{n} \uparrow \infty$ such that the following conditions are satisfied.

(a) Let $N_{i}$ be i.i.d. copies of $N$. Then

$$
\left(b_{n}^{-1} \sum_{i=1}^{n}\left(N_{i}\left(0, M_{n} t\right]-\lambda M_{n} t\right)\right)_{t \geq 0} \stackrel{\mathrm{FDD}}{\longrightarrow}(\xi(t))_{t \geq 0},
$$

where $(\xi(t))$ is some nondegenerate at 0 stochastic process.

(b) Let $\left(Z_{m}^{(i)}\right)_{m \in \mathbb{Z}}$ for $i \in \mathbb{N}$ be i.i.d. copies of $\left(Z_{m}\right)_{m \in \mathbb{Z}}$. Then

$$
b_{n}^{-1} \sum_{i=1}^{n} \sum_{m=1}^{\left\lfloor M_{n}\right\rfloor}\left(Z_{m}^{(i)}-\mathrm{E}(Z)\right) \stackrel{\mathrm{P}}{\rightarrow} 0 \quad \text { as } n \rightarrow \infty .
$$

(c) Let $I_{i}^{*}(0)$ be the total amount of data, of the ith input process, in the session arriving by time 0 that does not finish by that time. Then

$$
b_{n}^{-1} \sum_{i=1}^{n} I_{i}^{*}(0) \stackrel{\mathrm{P}}{\rightarrow} 0 \quad \text { as } n \rightarrow \infty .
$$

Under these conditions, the normalized process $S_{n}(t)=b_{n}^{-1} D_{n, M_{n}}(t), n \in \mathbb{N}, t \geq 0$, satisfies

$$
\left(S_{n}(t)\right)_{t \geq 0} \stackrel{\mathrm{FDD}}{\longrightarrow}(\mathrm{E}(Z) \xi(t))_{t \geq 0} \quad \text { as } n \rightarrow \infty .
$$

As we will see, the slow growth condition (3.2) is needed only for the verification of Theorem 3.2(c).

\section{Some renewal and extreme value theory}

Our first proposition in this section deals with the tails of randomly stopped random sums when both the individual terms and the number of terms have infinite means. It complements the existing results dealing with the situations where at least one of these means is finite; see, e.g. Faÿ et al. (2006). 
Proposition 4.1. Let $\left(X_{k}\right)$ be i.i.d. random variables independent of the positive integer-valued random variable $K$ with distribution function $F_{K}$, and let $T_{K}=\sum_{k=1}^{K} X_{k}$ with distribution function $F_{T_{K}}$. Let $G$ be the distribution function of $\left|X_{1}\right|$. Assume that

$$
\bar{F}_{K} \in \mathcal{R}_{-\kappa} \text { for some } 0<\kappa<1
$$

and that

$$
\bar{G} \in \mathcal{R}_{-\gamma} \text { for some } 0<\gamma<1, \quad \lim _{x \rightarrow \infty} \frac{\mathrm{P}\left(X_{1}>x\right)}{\bar{G}(x)}=p \in(0,1]
$$

Then

$$
\lim _{x \rightarrow \infty} \frac{\mathrm{P}\left(T_{K}>x\right)}{\mathrm{P}\left(K>\bar{G}(x)^{-1}\right)}=\mathrm{E}\left(\left(S_{\gamma}\right)_{+}^{\gamma \kappa}\right),
$$

where $S_{\gamma}$ is a strictly $\gamma$-stable random variable such that

$$
\mathrm{P}\left(S_{\gamma}>x\right) \sim p x^{-\gamma} \text { and } \mathrm{P}\left(S_{\gamma}<-x\right) \sim(1-p) x^{-\gamma} \text { as } x \rightarrow \infty .
$$

In particular, $\bar{F}_{T_{K}} \in \mathcal{R}_{-\kappa \gamma}$.

Proof. For $k \geq 1$, let $a_{k}:=\bar{G}^{\leftarrow}(1 / k)$, and note that

$$
\frac{1}{a_{k}}\left(X_{1}+\cdots+X_{k}\right) \stackrel{\mathrm{w}}{\rightarrow} S_{\gamma} \quad \text { as } k \rightarrow \infty
$$

(cf. (2.3)). For large $M>1$, we write

$$
\begin{aligned}
\mathrm{P}\left(T_{K}>x\right)= & \mathrm{P}\left(T_{K}>x, K>M \bar{G}(x)^{-1}\right)+\mathrm{P}\left(T_{K}>x, K \leq M^{-1} \bar{G}(x)^{-1}\right) \\
& +\mathrm{P}\left(T_{K}>x, M^{-1} \bar{G}(x)^{-1}<K \leq M \bar{G}(x)^{-1}\right) \\
= & : E_{1, M}(x)+E_{2, M}(x)+E_{3, M}(x) .
\end{aligned}
$$

Note that, as $x \rightarrow \infty$,

$$
E_{1, M}(x) \leq \mathrm{P}\left(K>M \bar{G}(x)^{-1}\right) \sim M^{-\kappa} \mathrm{P}\left(K>\bar{G}(x)^{-1}\right),
$$

and so

$$
\lim _{M \rightarrow \infty} \limsup _{x \rightarrow \infty} \frac{E_{1, M}(x)}{\mathrm{P}\left(K>\bar{G}(x)^{-1}\right)}=0 .
$$

Furthermore, we claim that, for any $\kappa<\kappa_{1}<1$ and all large enough $M$,

$$
\limsup _{x \rightarrow \infty} \frac{E_{2, M}(x)}{\mathrm{P}\left(K>\bar{G}(x)^{-1}\right)} \leq M^{-\left(1-\kappa_{1}\right)} .
$$

Indeed, suppose that (4.4) fails for some $\kappa<\kappa_{1}<1$. Then there is a sequence $x_{j} \uparrow \infty$ such that $j \bar{G}\left(x_{j}\right) \rightarrow 0$ as $j \rightarrow \infty$ and

$$
E_{2, j}\left(x_{j}\right) \geq \frac{1}{2} j^{-\left(1-\kappa_{1}\right)} \mathrm{P}\left(K>\bar{G}\left(x_{j}\right)^{-1}\right) \quad \text { for } j \in \mathbb{N} .
$$

Let $p_{k}:=\mathrm{P}(K=k)$ for $k \geq 1$. Note that

$$
E_{2, j}\left(x_{j}\right)=\sum_{k=1}^{\left\lfloor j^{-1} \bar{G}\left(x_{j}\right)^{-1}\right\rfloor} p_{k} \mathrm{P}\left(X_{1}+\cdots+X_{k}>x_{j}\right) .
$$


Theorem 9.1 of Denisov et al. (2008) shows that

$$
\mathrm{P}\left(X_{1}+\cdots+X_{k}>x_{j}\right) \sim k p \bar{G}\left(x_{j}\right)
$$

as $j \rightarrow \infty$ uniformly in $k \leq j^{-1} \bar{G}\left(x_{j}\right)^{-1}$. Therefore, for large $j$, by Karamata's theorem,

$$
E_{2, j}\left(x_{j}\right) \leq 2 \sum_{k=1}^{\left\lfloor j^{-1} \bar{G}\left(x_{j}\right)^{-1}\right\rfloor} k p_{k} p \bar{G}\left(x_{j}\right) \leq \frac{4 p}{1-\kappa} j^{-1} \mathrm{P}\left(K>j^{-1} \bar{G}\left(x_{j}\right)^{-1}\right),
$$

and by Potters's inequalities (cf. Resnick (2006, p. 36)), for any $\kappa<\kappa_{2}<\kappa_{1}$, there exists a $C_{1}>0$ such that, for large $j$,

$$
E_{2, j}\left(x_{j}\right) \leq C_{1} j^{-\left(1-\kappa_{2}\right)} \mathrm{P}\left(K>\bar{G}\left(x_{j}\right)^{-1}\right) .
$$

This clearly contradicts (4.5), and so (4.4) has to hold. We conclude that

$$
\lim _{M \rightarrow \infty} \limsup _{x \rightarrow \infty} \frac{E_{2, M}(x)}{\mathrm{P}\left(K>\bar{G}(x)^{-1}\right)}=0 .
$$

We now consider the term $E_{3, M}(x)$ in (4.2). For $M^{-1} \bar{G}(x)^{-1}<k \leq M \bar{G}(x)^{-1}$, we let $r=$ $x a_{k}^{-1}$. Since $\bar{G}\left(a_{k}\right) \sim k^{-1}$ as $k \rightarrow \infty$, we see that, for all large enough $x$, and $M^{-1} \bar{G}(x)^{-1}<$ $k \leq M \bar{G}(x)^{-1}$,

$$
(2 M)^{-1} \bar{G}(x)^{-1} \leq \bar{G}\left(a_{k}\right)^{-1} \leq 2 M \bar{G}(x)^{-1},
$$

which implies that, for the same range of $x$ and $k,(4 M)^{-1} \leq r^{\gamma} \leq 4 M$. In particular, $\mathrm{P}\left(S_{\gamma}>r\right)$ is bounded away from 0 . Since, by (4.1),

$$
\mathrm{P}\left(X_{1}+\cdots+X_{k}>x\right)=\mathrm{P}\left(\frac{1}{a_{k}}\left(X_{1}+\cdots+X_{k}\right)>r\right) \rightarrow \mathrm{P}\left(S_{\gamma}>r\right) \quad \text { as } k \rightarrow \infty
$$

(if $r$ is kept fixed), we conclude that

$$
\lim _{x \rightarrow \infty} \sup _{M^{-1} \bar{G}(x)^{-1}<k \leq M \bar{G}(x)^{-1}}\left|\frac{\mathrm{P}\left(X_{1}+\cdots+X_{k}>x\right)}{\mathrm{P}\left(S_{\gamma}>x / a_{k}\right)}-1\right|=0 .
$$

Therefore,

$$
E_{3, M}(x) \sim \sum_{k=\left\lfloor M^{-1} \bar{G}(x)^{-1}\right\rfloor+1}^{\left\lfloor M \bar{G}(x)^{-1}\right\rfloor} p_{k} \mathrm{P}\left(S_{\gamma}>\frac{x}{a_{k}}\right) \text { as } x \rightarrow \infty .
$$

If $f$ denotes the density of $S_{\gamma}$, this statement translates by Fubini into

$$
\begin{aligned}
E_{3, M}(x) & \sim \int_{0}^{\infty} \sum_{k=\left\lfloor M^{-1} \bar{G}(x)^{-1}\right\rfloor+1}^{\left\lfloor M \bar{G}(x)^{-1}\right\rfloor} p_{k} \mathbf{1}_{\left\{a_{k}>x / y\right\}} f(y) \mathrm{d} y \\
& \sim \int_{0}^{\infty} \sum_{k=\left\lfloor M^{-1} \bar{G}(x)^{-1}\right\rfloor+1}^{\left\lfloor M \bar{G}(x)^{-1}\right\rfloor} p_{k} \mathbf{1}_{\left\{k>\bar{G}(x / y)^{-1}\right\}} f(y) \mathrm{d} y \\
& =\int_{0}^{\infty}\left[\mathrm{P}\left(\max \left(M^{-1} \bar{G}(x)^{-1}, \bar{G}\left(\frac{x}{y}\right)^{-1}\right)<K \leq M \bar{G}(x)^{-1}\right)\right] f(y) \mathrm{d} y .
\end{aligned}
$$


Now, for every $y>0$, as $x \rightarrow \infty$,

$$
\frac{\mathrm{P}\left(\max \left(M^{-1} \bar{G}(x)^{-1}, \bar{G}(x / y)^{-1}\right)<K \leq M \bar{G}(x)^{-1}\right)}{\mathrm{P}\left(K>\bar{G}(x)^{-1}\right)} \rightarrow\left[\min \left(M^{\kappa}, y^{\gamma \kappa}\right)-M^{-\kappa}\right]_{+},
$$

while the same ratio on the left-hand side is bounded from above for large $x$ uniformly in $y>0$ by

$$
\frac{\mathrm{P}\left(K>M^{-1} \bar{G}(x)^{-1}\right)}{\mathrm{P}\left(K>\bar{G}(x)^{-1}\right)} \leq 2 M^{\kappa}
$$

Therefore, by the dominated convergence theorem,

$$
\lim _{x \rightarrow \infty} \frac{E_{3, M}(x)}{\mathrm{P}\left(K>\bar{G}(x)^{-1}\right)}=\int_{0}^{\infty}\left[\min \left(M^{\kappa}, y^{\gamma \kappa}\right)-M^{-\kappa}\right]_{+} f(y) \mathrm{d} y .
$$

As $M \rightarrow \infty$, the right-hand side of (4.7) converges to $\mathrm{E}\left(\left(S_{\gamma}^{+}\right)^{\gamma \kappa}\right)$, and so the statement of the proposition follows from (4.2), (4.3), (4.6), and (4.7).

The next two results are renewal theorems needed in the proof of the main theorem.

Proposition 4.2. Let $\left(X_{k}\right)$ be an i.i.d. sequence of positive random variables with distribution function $F$, such that $\bar{F} \in \mathcal{R}_{-1 / \beta}, 0<1 / \beta<1$. Let $T_{j}=\sum_{k=1}^{j} X_{k}, j \in \mathbb{N}_{0}$. Suppose that $(c(t))_{t \geq 0}$ is a nonnegative eventually nonincreasing function, regularly varying with index $-\eta$ at $\infty, 1<\eta<2$. Then

$$
\sum_{j=0}^{\infty} c(j) \mathrm{P}\left(T_{j}>x\right) \sim \frac{1}{\eta-1} C_{\eta, \beta} \bar{F}(x)^{-1} c\left(\bar{F}(x)^{-1}\right) \in \mathcal{R}_{(1-\eta) / \beta} \quad \text { as } x \rightarrow \infty,
$$

where $C_{\eta, \beta}=\mathrm{E}\left(\left(S_{1 / \beta}(1)\right)^{(\eta-1) / \beta}\right)$ and $S_{1 / \beta}$ is the positive, strictly $1 / \beta$-stable stochastic process in (2.3).

Proof. Let $H_{\beta}$ be the distribution function of $S_{1 / \beta}(1)$. Then, by the weak convergence in $(2.3)$,

$$
\lim _{n \rightarrow \infty} \sup _{r \in \mathbb{R}}\left|H_{\beta}(r)-\mathrm{P}\left(T_{n} \leq a_{n} r\right)\right|=0,
$$

where $a_{n}=\bar{F}^{\leftarrow}(1 / n)$ (cf. Petrov (1975, Theorem 11, p. 15, and Theorem 10, p. 88)). Thus, there exists a positive sequence $\left(\varepsilon_{j}\right)_{j \geq 0}$ with $\varepsilon_{j} \downarrow 0$ as $j \rightarrow \infty$ such that, for any $r>0$,

$$
\mathrm{P}\left(T_{j}>r\right) \leq \bar{H}_{\beta}\left(a_{j}^{-1} r\right)+\varepsilon_{j} .
$$

Let $\delta_{1}, \delta_{2}>0, \delta_{1}<\delta_{2}$, and $\delta=\left(\delta_{1}, \delta_{2}\right)$. Then

$$
\begin{aligned}
\sum_{j=\left\lceil\delta_{1} \bar{F}(r)^{-1}\right\rceil}^{\left\lfloor\delta_{2} \bar{F}(r)^{-1}\right\rfloor} c(j) \mathrm{P}\left(T_{j}>r\right) & \leq \sum_{j=\left\lceil\delta_{1} \bar{F}(r)^{-1}\right\rceil}^{\left\lfloor\delta_{2} \bar{F}(r)^{-1}\right\rfloor} c(j) \bar{H}_{\beta}\left(a_{j}^{-1} r\right)+\sum_{j=\left\lceil\delta_{1} \bar{F}(r)^{-1}\right\rceil}^{\left\lfloor\delta_{2} \bar{F}(r)^{-1}\right\rfloor} c(j) \varepsilon_{j} \\
& =: J_{1}(\delta, r)+J_{2}(\delta, r) .
\end{aligned}
$$

We begin by studying the first summand. Let $x_{j}^{(r)}:=j \bar{F}(r)$, and let $\ell$ be a slowly varying function such that $\bar{F}(x)=\ell(x) x^{-1 / \beta}$. Then, as $n \rightarrow \infty$,

$$
n \bar{F}\left(a_{n}\right)=n \ell\left(a_{n}\right) a_{n}^{-1 / \beta} \rightarrow 1 .
$$


Since $\delta_{1} \bar{F}(r)^{-1} \leq j \leq \delta_{2} \bar{F}(r)^{-1}$, we have, for some $C_{1}, C_{2}>0$ and all large enough $r$,

$$
C_{1} r \leq \bar{F}^{\leftarrow}(j)=a_{j} \leq C_{2} r .
$$

By Theorem 1.5.2 of Bingham et al. (1987), we obtain $\ell\left(a_{j}\right) \sim \ell(r)$ as $r \rightarrow \infty$ uniformly for $\delta_{1} \bar{F}(r)^{-1} \leq j \leq \delta_{2} \bar{F}(r)^{-1}$. Thus, (4.8) gives $\ell(r) \sim j^{-1} a_{j}^{1 / \beta}$ as $r \rightarrow \infty$ uniformly for $\delta_{1} \bar{F}(r)^{-1} \leq j \leq \delta_{2} \bar{F}(r)^{-1}$, and

$$
\left(x_{j}^{(r)}\right)^{-\beta}=\left(j \ell(r) r^{-1 / \beta}\right)^{-\beta} \sim a_{j}^{-1} r \quad \text { as } r \rightarrow \infty .
$$

Hence, as $r \rightarrow \infty$,

$$
\begin{aligned}
\sum_{j=\left\lceil\delta_{1} \bar{F}(r)^{-1}\right\rceil}^{\left\lfloor\delta_{2} \bar{F}(r)^{-1}\right\rfloor} c(j) \bar{H}_{\beta}\left(a_{j}^{-1} r\right) & \sim \sum_{j=\left\lceil\delta_{1} \bar{F}(r)^{-1}\right\rceil}^{\left\lfloor\delta_{2} \bar{F}(r)^{-1}\right\rfloor} c\left(x_{j}^{(r)} \bar{F}(r)^{-1}\right) \bar{H}_{\beta}\left(\left(x_{j}^{(r)}\right)^{-\beta}\right) \\
& =\bar{F}(r)^{-1} \sum_{j=\left\lceil\delta_{1} \bar{F}(r)^{-1}\right\rceil}^{\left\lfloor\delta_{2} \bar{F}(r)^{-1}\right\rfloor}\left(x_{j+1}^{(r)}-x_{j}^{(r)}\right) c\left(x_{j}^{(r)} \bar{F}(r)^{-1}\right) \bar{H}_{\beta}\left(\left(x_{j}^{(r)}\right)^{-\beta}\right) .
\end{aligned}
$$

Since $c \in \mathcal{R}_{-\eta}$, we obtain, by Theorem 1.5.2 of Bingham et al. (1987), as $r \rightarrow \infty$,

$$
\begin{aligned}
& \frac{1}{\bar{F}(r)^{-1} c\left(\bar{F}(r)^{-1}\right)} \sum_{j=\left\lceil\delta_{1} \bar{F}(r)^{-1}\right\rceil}^{\left\lfloor\delta_{2} \bar{F}(r)^{-1}\right\rfloor} c(j) \bar{H}_{\beta}\left(a_{j}^{-1} r\right) \\
& \sim \sum_{j=\left\lceil\delta_{1} \bar{F}(r)^{-1}\right\rceil}^{\left\lfloor\delta_{2} \bar{F}(r)^{-1}\right\rfloor}\left(x_{j+1}^{(r)}-x_{j}^{(r)}\right)\left(x_{j}^{(r)}\right)^{-\eta} \bar{H}_{\beta}\left(\left(x_{j}^{(r)}\right)^{-\beta}\right) \\
& \sim \int_{\delta_{1}}^{\delta_{2}} y^{-\eta} \bar{H}_{\beta}\left(y^{-\beta}\right) \mathrm{d} y,
\end{aligned}
$$

and so

$$
J_{1}(\delta, r) \sim \bar{F}(r)^{-1} c\left(\bar{F}(r)^{-1}\right) \int_{\delta_{1}}^{\delta_{2}} y^{-\eta} \bar{H}_{\beta}\left(y^{-\beta}\right) \text { d } y \quad \text { as } r \rightarrow \infty .
$$

On the other hand,

$$
\begin{aligned}
J_{2}(\delta, r) & \leq \varepsilon_{\delta_{1} \bar{F}(r)^{-1}} \sum_{j \geq \delta_{1} \bar{F}(r)^{-1}} c(j) \\
& \sim \varepsilon_{\delta_{1} \bar{F}(r)^{-1}}(\eta-1)^{-1} \delta_{1} \bar{F}(r)^{-1} c\left(\delta_{1} \bar{F}(r)^{-1}\right) \\
& \sim \varepsilon_{\delta_{1} \bar{F}(r)^{-1}}(\eta-1)^{-1} \delta_{1}^{1-\eta} \bar{F}(r)^{-1} c\left(\bar{F}(r)^{-1}\right) \quad \text { as } r \rightarrow \infty
\end{aligned}
$$

by Bingham et al. (1987, Proposition 1.5.10). Since $\delta_{1}$ is arbitrary and $\varepsilon_{\delta_{1}} \bar{F}(r)^{-1} \rightarrow 0$ as $r \rightarrow \infty$, we obtain

$$
\lim _{\delta_{2} \rightarrow \infty} \lim _{\delta_{1} \downarrow 0} \lim _{r \rightarrow \infty} \bar{F}(r) c\left(\bar{F}(r)^{-1}\right)^{-1}\left(J_{1}(\delta, r)+J_{2}(\delta, r)\right)=\frac{1}{\eta-1} \mathrm{E}\left(\left(S_{1 / \beta}(1)\right)^{(\eta-1) / \beta}\right) .
$$


Next, Proposition 1.5.8 of Bingham et al. (1987) and Lemma 6.4 result in

$$
\begin{aligned}
\sum_{j \leq \delta_{1} \bar{F}(r)^{-1}} c(j) \mathrm{P}\left(T_{j}>r\right) & \leq C_{3} \sum_{j \leq \delta_{1} \bar{F}(r)^{-1}} c(j) j \bar{F}(r) \\
& \sim C_{4} \delta_{1}^{2-\eta} \bar{F}(r)^{-1} c\left(\bar{F}(r)^{-1}\right) \quad \text { as } r \rightarrow \infty
\end{aligned}
$$

for some $C_{3}, C_{4}>0$. Hence,

$$
\lim _{\delta_{1} \downarrow 0} \lim _{r \rightarrow \infty} \bar{F}(r) c\left(\bar{F}(r)^{-1}\right)^{-1} \sum_{j \leq \delta_{1} \bar{F}(r)^{-1}} c(j) \mathrm{P}\left(T_{j}>r\right)=0 .
$$

Also, by Bingham et al. (1987, Proposition 1.5.10),

$$
\begin{aligned}
\bar{F}(r) c\left(\bar{F}(r)^{-1}\right)^{-1} \sum_{j \geq \delta_{2} \bar{F}(r)^{-1}} c(j) \mathrm{P}\left(T_{j}>r\right) & \leq \bar{F}(r) c\left(\bar{F}(r)^{-1}\right)^{-1} \sum_{j \geq \delta_{2} \bar{F}(r)^{-1}} c(j) \\
& \sim \frac{1}{\eta-1} \delta_{2}^{1-\eta} \\
& \rightarrow 0 \quad \text { as } \delta_{2} \rightarrow \infty
\end{aligned}
$$

By (4.9), (4.10), and (4.11), the result follows.

The following result is a local renewal theorem.

Lemma 4.1. Let the conditions of Proposition 4.2 hold, and assume additionally that Assumption 2.2 is satisfied. Then

$$
\begin{aligned}
& \sum_{j=0}^{\infty} c(j)\left[\mathrm{P}\left(T_{j}>x\right)-\mathrm{P}\left(T_{j}>x+1\right)\right] \\
& \quad \sim \frac{1}{\beta} C_{\eta, \beta} x^{-1} \bar{F}(x)^{-1} c\left(\bar{F}(x)^{-1}\right) \in \mathcal{R}_{(1-\eta) / \beta-1} \text { as } x \rightarrow \infty .
\end{aligned}
$$

Proof. Under the first scenario of Assumption 2.2, the proof, using Proposition 4.2, is the same as the proof of Theorem 2 of Anderson and Athreya (1988), which in particular requires that $\beta<2$. Under the second scenario of Assumption 2.2, the statement is Theorem 3 of Doney (1997).

\section{Verification of the conditions of Theorem 3.2}

The main result of this paper, Theorem 3.1, is proved in this section by verifying the conditions of Theorem 3.2.

\subsection{Verification of Theorem 3.2(a)}

Proposition 5.1. Let the Poisson cluster model satisfy Assumptions 2.1 and 2.2. Furthermore, let $M_{n}$ and $b_{n}$ be sequences of positive constants such that $M_{n} \uparrow \infty$ and $b_{n} \uparrow \infty$. Let $N_{i}$ be i.i.d. copies of $N$. Then

$$
\left(b_{n}^{-1} \sum_{i=1}^{n}\left(N_{i}\left(0, M_{n} t\right]-\lambda M_{n} t\right)\right)_{t \geq 0} \stackrel{\mathrm{FDD}}{\longrightarrow}\left(B_{H}(t)\right)_{t \geq 0},
$$

where $\left(B_{H}(t)\right)_{t \geq 0}$ is as given in Theorem 3.1. 
Proof. We can write

$$
\begin{aligned}
b_{n}^{-1} \sum_{i=1}^{n}\left[N_{i}\left(0, M_{n} t\right]-\lambda M_{n} t\right]= & b_{n}^{-1} \sum_{i=1}^{n}\left[N_{i}^{\left(0, M_{n} t\right]}\left(0, M_{n} t\right]-\mathrm{E}\left(N_{i}^{\left(0, M_{n} t\right]}\left(0, M_{n} t\right]\right)\right] \\
& +b_{n}^{-1} \sum_{i=1}^{n}\left[N_{i}^{(-\infty, 0]}\left(0, M_{n} t\right]-\mathrm{E}\left(N_{i}^{(-\infty, 0]}\left(0, M_{n} t\right]\right)\right] \\
= & : \xi_{n}^{+}(t)+\xi_{n}^{-}(t),
\end{aligned}
$$

where $N_{i}^{A}(B)=\#\left\{\Gamma_{m, k}^{(i)}: m \in \mathbb{Z}, k \in\left\{0, \ldots, K_{m}^{(i)}\right\}, \Gamma_{m, k}^{(i)}=\Gamma_{m}^{(i)}+T_{m, k}^{(i)} \in B\right.$ and $\left.\Gamma_{m}^{(i)} \in A\right\}$. We will show in Lemma 5.1 and Lemma 5.2, below, that

$$
\left(\xi_{n}^{+}(t)\right)_{t \geq 0} \stackrel{\mathrm{FDD}}{\longrightarrow}\left(B_{H}^{+}(t)\right)_{t \geq 0} \quad \text { and } \quad\left(\xi_{n}^{-}(t)\right)_{t \geq 0} \stackrel{\mathrm{FDD}}{\longrightarrow}\left(B_{H}^{-}(t)\right)_{t \geq 0},
$$

where $\left(B_{H}^{+}(t)\right)_{t \geq 0}$ and $\left(B_{H}^{-}(t)\right)_{t \geq 0}$ are independent fractional Brownian motions of index $H$ with time 1 variances

$$
\sigma_{+}^{2}=\frac{2 \lambda_{0}}{2+\beta-\alpha} \int_{0}^{\infty} y^{-(2+\beta-\alpha) / \beta} \mathrm{P}\left(S_{1 / \beta}(1) \leq y\right) \mathrm{d} y
$$

and

$$
\begin{aligned}
\sigma_{-}^{2}=\lambda_{0} \int_{0}^{\infty} \mathrm{E}( & \frac{2}{2-\alpha} I(w+1)^{2-\alpha} \\
& \left.+\frac{2}{\alpha-1} I(w) I(w+1)^{1-\alpha}-\frac{2}{(2-\alpha)(\alpha-1)} I(w)^{2-\alpha}\right) \mathrm{d} w
\end{aligned}
$$

By the independence of $\left(\xi_{n}^{+}(t)\right)$ and $\left(\xi_{n}^{-}(t)\right),(5.1)$ implies that

$$
\left(\xi_{n}(t)\right)_{t \geq 0}:=\left(\xi_{n}^{+}(t)+\xi_{n}^{-}(t)\right)_{t \geq 0} \stackrel{\mathrm{FDD}}{\longrightarrow}\left(B_{H}^{+}(t)+B_{H}^{-}(t)\right)_{t \geq 0}=:\left(B_{H}(t)\right)_{t \geq 0},
$$

where $\left(B_{H}(t)\right)_{t \geq 0}$ is a fractional Brownian motion with time 1 variance $\sigma^{2}=\sigma_{+}^{2}+\sigma_{-}^{2}$.

In order to prove (5.1), we note that $\xi_{n}^{+}(t)$ and $\xi_{n}^{-}(t)$ are infinitely divisible random variables whose characteristic functions can be written in the form

$$
\mathrm{E}\left(\exp \left(\mathrm{i} \theta \xi_{n}^{ \pm}(t)\right)\right)=\exp \left\{\int_{0}^{\infty}\left(\mathrm{e}^{\mathrm{i} \theta x}-1-\mathrm{i} \theta x\right) v_{n, t}^{ \pm}(\mathrm{d} x)\right\},
$$

where $v_{n, t}^{ \pm}$are the corresponding Lévy measures. These can be represented in the form

$$
v_{n, t}^{ \pm}=n \lambda_{0}\left(\mathrm{P}_{1} \times \mathrm{Leb}\right) \circ \zeta_{ \pm}^{-1}
$$

with the following notation. Let $\left(\Omega_{1}, \mathcal{F}_{1}, \mathrm{P}_{1}\right)$ be a probability space on which a generic cluster process $\left(N_{c}[0, u]\right)_{u \geq 0}$ is defined. The maps $\zeta_{+}$and $\zeta_{-}$are defined as follows: $\zeta_{+}: \Omega_{1} \times$ $\left(0, M_{n} t\right] \rightarrow[0, \infty)$ is given by $\zeta_{+}\left(\omega_{1}, u\right)=N_{c}[0, u]\left(\omega_{1}\right) / b_{n}$ and $\zeta_{-}: \Omega_{1} \times \mathbb{R}_{+} \rightarrow[0, \infty)$ is given by $\zeta_{-}\left(\omega_{1}, u\right)=N_{c}\left(u, u+M_{n} t\right]\left(\omega_{1}\right) / b_{n}$. To see this, write $N_{i}^{\left(0, M_{n} t\right]}\left(0, M_{n} t\right]$ and $N_{i}^{(-\infty, 0]}\left(0, M_{n} t\right]$ as integrals with respect to a Poisson random measure and use, for example, 
Lemma 12.2(i) of Kallenberg (2002) (cf. the proof of Proposition 3.5 of Faÿ et al. (2006)). For notational simplicity, below we often drop the subscript in $\mathrm{P}_{1}$ and, hence, write, for $A \in \mathscr{B}(\mathbb{R})$,

$$
\begin{aligned}
& v_{n, t}^{+}(A)=n \lambda_{0} \int_{0}^{M_{n} t} \mathrm{P}\left(\frac{N_{c}[0, u]}{b_{n}} \in A\right) \mathrm{d} u, \\
& v_{n, t}^{-}(A)=n \lambda_{0} \int_{0}^{\infty} \mathrm{P}\left(\frac{N_{c}\left(u, u+M_{n} t\right]}{b_{n}} \in A\right) \mathrm{d} u .
\end{aligned}
$$

Since the Lévy measures are concentrated on the positive half-line, we can apply standard results for the weak convergence of infinitely divisible distributions; see, e.g. Theorem 15.14 of Kallenberg (2002). Without loss of generality, we will assume that $\lambda_{0}=1$ in the following.

Lemma 5.1. Let Assumption 2.1 hold, and let $t \geq 0$ and $\varepsilon>0$. Then

(a) $v_{n, t}^{+} \stackrel{\mathrm{v}}{\rightarrow} 0$ on $(0, \infty]$ as $n \rightarrow \infty$;

(b) $\lim _{n \rightarrow \infty} \int_{\{|x| \leq \varepsilon\}} x^{2} v_{n, t}^{+}(\mathrm{d} x)=t^{2 H} \sigma_{+}^{2}$ with $\sigma_{+}^{2}$ as in (5.2) and $H$ as in (3.3);

(c) $\lim _{n \rightarrow \infty} \int_{\{|x|>\varepsilon\}} x v_{n, t}^{+}(\mathrm{d} x)=0$.

In particular,

$$
\left(\xi_{n}^{+}(t)\right)_{t \geq 0} \stackrel{\mathrm{FDD}}{\longrightarrow}\left(B_{H}^{+}(t)\right)_{t \geq 0},
$$

where $\left(B_{H}^{+}(t)\right)_{t \geq 0}$ is a fractional Brownian motion with Hurst index $H$ and time 1 variance $\sigma_{+}^{2}$.

Proof. We use the decompositions

$$
\begin{aligned}
\int_{\{|x| \leq \varepsilon\}} x^{2} v_{n, t}^{+}(\mathrm{d} x)= & \frac{n}{b_{n}^{2}} \mathrm{E}\left(\mathbf{1}_{\left\{K+1 \leq \varepsilon b_{n}\right\}} \int_{0}^{M_{n} t} N_{c}[0, u]^{2} \mathrm{~d} u\right) \\
& +\frac{n}{b_{n}^{2}} \mathrm{E}\left(\mathbf{1}_{\left\{K+1>\varepsilon b_{n}\right\}} \int_{0}^{M_{n} t \wedge T_{\left\lfloor\varepsilon b_{n}-1\right\rfloor}} N_{c}[0, u]^{2} \mathrm{~d} u\right) \\
= & : I_{1,1}(n)+I_{1,2}(n)
\end{aligned}
$$

and

$$
\begin{aligned}
\int_{\{|x|>\varepsilon\}} x v_{n, t}^{+}(\mathrm{d} x) & =n \varepsilon \int_{0}^{M_{n} t} \mathrm{P}\left(N_{c}[0, u]>\varepsilon b_{n}\right) \mathrm{d} u+n \int_{0}^{M_{n} t} \int_{\varepsilon}^{\infty} \mathrm{P}\left(N_{c}[0, u]>x b_{n}\right) \mathrm{d} x \mathrm{~d} u \\
& =: I_{2,1}(n)+I_{2,2}(n) .
\end{aligned}
$$

Claim (b) now follows from Lemma 6.5 and Lemma 6.6, below, while claim (c) follows from Lemma 6.7 and Lemma 6.8, below. Then (a) is a conclusion of

$$
0 \leq \limsup _{n \rightarrow \infty} v_{n, t}^{+}(\varepsilon, \infty) \leq \limsup _{n \rightarrow \infty} \varepsilon^{-1} \int_{\{|x|>\varepsilon\}} x v_{n, t}^{+}(\mathrm{d} x)=0 \quad \text { for all } \varepsilon>0 .
$$

Hence, (a)-(c) and Theorem 15.14 of Kallenberg (2002) result in

$$
\xi_{n}^{+}(t) \stackrel{\mathrm{w}}{\rightarrow} B_{H}^{+}(t) \quad \text { as } n \rightarrow \infty \text { for all } t \geq 0 .
$$

Applying Lemma 4.8 of Kallenberg (2002) we see that, for every $k \geq 1$ and $0 \leq t_{1}<t_{2}<$ $\cdots<t_{k}<\infty$, the family of laws of the random vectors

$$
\left(\xi_{n}^{+}\left(t_{1}\right), \ldots, \xi_{n}^{+}\left(t_{k}\right)\right)_{n \in \mathbb{N}}
$$


are tight. Let $\tilde{\boldsymbol{B}}_{H}=\left(\tilde{B}_{H}\left(t_{1}\right), \ldots, \tilde{B}_{H}\left(t_{k}\right)\right)$ be a weak subsequential limit of this family, i.e. there exists a subsequence $\left(n_{i}\right)$ such that

$$
\left(\xi_{n_{i}}^{+}\left(t_{1}\right), \ldots, \xi_{n_{i}}^{+}\left(t_{k}\right)\right) \rightarrow \tilde{\boldsymbol{B}}_{H} \quad \text { as } i \rightarrow \infty
$$

On the one hand, $\tilde{\boldsymbol{B}}_{H}$ is infinitely divisible (because of the Poisson arrivals of clusters). On the other hand, the one-dimensional marginal distributions of $\tilde{\boldsymbol{B}}_{H}$ are Gaussian with $\tilde{B}_{H}\left(t_{i}\right) \stackrel{\mathrm{D}}{=} B_{H}^{+}\left(t_{i}\right)$ by (5.6). Hence, $\tilde{\boldsymbol{B}}_{H}$ is zero-mean multivariate Gaussian. We will now compute its covariance matrix. The stationarity of the $N_{i}^{+}$s and, hence, that of the $\xi_{n}^{+}$s imply by (5.6) that, for $1 \leq j \leq i \leq k$,

$$
\xi_{n}^{+}\left(t_{i}\right)-\xi_{n}^{+}\left(t_{j}\right) \stackrel{\mathrm{w}}{\rightarrow} B_{H}^{+}\left(t_{i}-t_{j}\right) \quad \text { as } n \rightarrow \infty .
$$

Thus, $\tilde{B}_{H}\left(t_{i}\right)-\tilde{B}_{H}\left(t_{j}\right) \stackrel{\mathrm{D}}{=} B_{H}^{+}\left(t_{i}-t_{j}\right)$, and so

$$
\begin{aligned}
\operatorname{cov}\left(\tilde{B}_{H}\left(t_{i}\right), \tilde{B}_{H}\left(t_{j}\right)\right) & =\frac{1}{2}\left(\mathrm{E}\left(\tilde{B}_{H}\left(t_{i}\right)^{2}\right)+\mathrm{E}\left(\tilde{B}_{H}\left(t_{j}\right)^{2}\right)-\mathrm{E}\left(\left(\tilde{B}_{H}\left(t_{i}\right)-\tilde{B}_{H}\left(t_{j}\right)\right)^{2}\right)\right) \\
& =\frac{1}{2}\left(\mathrm{E}\left(B_{H}^{+}\left(t_{i}\right)^{2}\right)+\mathrm{E}\left(B_{H}^{+}\left(t_{j}\right)^{2}\right)-\mathrm{E}\left(B_{H}^{+}\left(t_{i}-t_{j}\right)^{2}\right)\right) \\
& =\frac{\sigma_{+}^{2}}{2}\left(t_{i}^{2 H}+t_{j}^{2 H}-\left(t_{i}-t_{j}\right)^{2 H}\right) .
\end{aligned}
$$

This implies that the random vectors in (5.7) converge weakly to the corresponding finitedimensional distributions of the appropriate fractional Brownian motion, and this verifies the statement.

Lemma 5.2. Let Assumptions 2.1 and 2.2 hold, and let $t \geq 0$ and $\varepsilon>0$. Then

(a) $v_{n, t}^{-} \stackrel{\mathrm{v}}{\rightarrow} 0$ on $(0, \infty]$ as $n \rightarrow \infty$;

(b) $\lim _{n \rightarrow \infty} \int_{\{|x| \leq \varepsilon\}} x^{2} v_{n, t}^{-}(\mathrm{d} x)=t^{2 H} \sigma_{-}^{2}$ with $\sigma_{-}^{2}$ as in (5.3) and $H$ as in (3.3);

(c) $\lim _{n \rightarrow \infty} \int_{\{|x|>\varepsilon\}} x v_{n, t}^{-}(\mathrm{d} x)=0$.

In particular,

$$
\left(\xi_{n}^{-}(t)\right)_{t \geq 0} \stackrel{\mathrm{FDD}}{\longrightarrow}\left(B_{H}^{-}(t)\right)_{t \geq 0},
$$

where $\left(B_{H}^{-}(t)\right)_{t \geq 0}$ is a fractional Brownian motion with Hurst index $H$ and time 1 variance $\sigma_{-}^{2}$.

Proof. (b) The argument is similar to that of Lemma 5.1, but somewhat more involved technically. We start by introducing some notation. Let

$$
\begin{aligned}
& H_{n}^{(1)}(w):=\mathrm{E}\left(N_{c}\left(M_{n} w, M_{n}(w+t)\right]^{2} \mathbf{1}_{\left\{N_{c}\left(M_{n} w, M_{n}(w+t)\right] \leq \varepsilon b_{n}\right\}} \mathbf{1}_{\left\{K>N_{0}\left(0, M_{n}(w+t)\right]\right\}}\right), \\
& H_{n}^{(2)}(w):=\mathrm{E}\left(N_{c}\left(M_{n} w, M_{n}(w+t)\right]^{2} \mathbf{1}_{\left\{N_{c}\left(M_{n} w, M_{n}(w+t)\right] \leq \varepsilon b_{n}\right\}} \mathbf{1}_{\left\{N_{0}\left(0, M_{n} w\right]<K \leq N_{0}\left(0, M_{n}(w+t)\right]\right\}}\right),
\end{aligned}
$$

so that

$$
\mathrm{E}\left(N_{c}\left(M_{n} w, M_{n}(w+t)\right]^{2} \mathbf{1}_{\left\{N_{c}\left(M_{n} w, M_{n}(w+t)\right] \leq \varepsilon b_{n}\right\}}\right)=H_{n}^{(1)}(w)+H_{n}^{(2)}(w) .
$$


By Lemma 6.9, Lemma 6.10, and Theorem 6.1, we can use the dominated convergence theorem so that

$$
\begin{aligned}
\lim _{n \rightarrow \infty} \int_{\{|x| \leq \varepsilon\}} x^{2} v_{n, t}^{-}(\mathrm{d} x)= & \int_{0}^{\infty} \lim _{n \rightarrow \infty} \frac{n M_{n}}{b_{n}^{2}}\left(H_{n}^{(1)}(w)+H_{n}^{(2)}(w)\right) \mathrm{d} w \\
= & \int_{0}^{\infty}\left[\mathrm{E}\left((I(w+t)-I(w))^{2} I(w+t)^{-\alpha}\right)\right. \\
& +\mathrm{E}\left(\frac{\alpha}{2-\alpha} I(w+t)^{2-\alpha}+\frac{2 \alpha}{\alpha-1} I(w) I(w+t)^{1-\alpha}\right) \\
& \left.-\mathrm{E}\left(I(w)^{2} I(w+t)^{-\alpha}+\frac{2}{(2-\alpha)(\alpha-1)} I(w)^{2-\alpha}\right)\right] \mathrm{d} w .
\end{aligned}
$$

Now, by substituting $w$ for $t z$ and using the self-similarity of $I$ of index $1 / \beta$ we obtain (b).

(c) Let $M>0$. Then

$$
\begin{aligned}
\int_{\{|x|>\varepsilon\}} x v_{n, t}^{-}(\mathrm{d} x)= & \frac{n M_{n}}{b_{n}} \int_{M}^{\infty} \mathrm{E}\left(N_{c}\left(M_{n} w, M_{n}(w+t)\right] \mathbf{1}_{\left\{N_{c}\left(M_{n} w, M_{n}(w+t)\right]>\varepsilon b_{n}\right\}}\right) \mathrm{d} w \\
& +\frac{n M_{n}}{b_{n}} \int_{0}^{M} \mathrm{E}\left(N_{c}\left(M_{n} w, M_{n}(w+t)\right] \mathbf{1}_{\left\{N_{c}\left(M_{n} w, M_{n}(w+t)\right]>\varepsilon b_{n}\right\}}\right) \mathrm{d} w \\
= & : I_{3,1}(n)+I_{3,2}(n) .
\end{aligned}
$$

Therefore, (c) follows from Lemma 6.11, below. The remainder of the proof is the same as in Lemma 5.1.

\subsection{Verification of Theorem $3.2(b)$}

This is an immediate consequence of the Chebyshev inequality and (6.2), below.

\subsection{Verification of Theorem 3.2(c)}

It is in this part of the argument that the slow growth condition (3.2) plays a role. Condition (c) of Theorem 3.2 is a direct conclusion of (3.2) and the next lemma, since then $\lim _{n \rightarrow \infty} n \mathrm{P}\left(I^{*}(0)>b_{n}\right)=0$.

Lemma 5.3. Let the Poisson cluster model satisfy Assumption 2.1. Then there exists a constant $C>0$ such that

$$
\mathrm{P}\left(I^{*}(0)>z\right) \leq C z^{-\left(\alpha_{1}-1\right) / \beta_{2}} \text { for all } z>0 .
$$

Proof. The initial step is to show that we may, without loss of generality, assume that the interarrival times of the cluster process $N_{0}$ are bounded from below by a positive number. To this end, we modify the renewal point process $N_{0}$ into a different renewal point process, $\tilde{N}_{0}$, as follows. Let $\delta>0$ be such that $\mathrm{P}(X \geq \delta)>0$.

Let $\tilde{T}_{1}:=\min \left\{T_{j}: T_{j} \geq \delta\right\}$. Define $\tilde{Z}_{0}:=Z_{0}+\sum_{i=1}^{U_{1}-1}\left(Z_{i}+\delta\right)$, where $U_{1}:=\min \{j:$ $\left.T_{j} \geq \delta\right\}$. We view $\tilde{Z}_{0}$ as the amount of data in the single arrival at time $\tilde{T}_{0}:=0$.

In general, given $\tilde{T}_{m}$ and $U_{m}$, we define the next arrival by $\tilde{T}_{m+1}:=\min \left\{T_{j}: T_{j}-\tilde{T}_{m} \geq \delta\right\}$ and the amount of data brought in by the arrival at time $\tilde{T}_{m}$ as $\tilde{Z}_{m}:=Z_{U_{m}}+\sum_{i=U_{m}+1}^{U_{m+1}-1}\left(Z_{i}+\delta\right)$, where $U_{m+1}:=\min \left\{j: T_{j}-\tilde{T}_{m} \geq \delta\right\}$.

Note that with this (sample path) modification, every arrival point of the original process $N_{0}$ will arrive, in the new process, not later than before (but it may be aggregated with other points of $N_{0}$ into a single new arrival), and its transmission will last in the new process for at least as 
long as in the original process. We will still take a cluster of size $K$, so this modification can only increase the random variable $I^{*}(0)$.

For the new process, the random amount of data brought in with any arrival has the representation $\tilde{Z}_{0}=Z_{0}+\sum_{i=1}^{U_{1}-1}\left(Z_{i}+\delta\right)$, and since $U_{1}$ is stochastically dominated by a geometric random variable, we see that $\mathrm{E}\left(\tilde{Z}_{0}^{2}\right)<\infty$. Furthermore, the interarrival times of the new process satisfy $\tilde{X}_{i} \geq \delta$ almost surely and $\mathrm{P}\left(X_{1}>x\right) \leq \mathrm{P}\left(\tilde{X}_{i}>x\right) \leq \mathrm{P}\left(X_{1}+\delta>x\right) \sim \mathrm{P}\left(X_{1}>x\right)$ as $x \rightarrow \infty$. Hence, $\mathrm{P}\left(\tilde{X}_{1}>x\right) \sim \mathrm{P}\left(X_{1}>x\right)$ as $x \rightarrow \infty$. Therefore, for the purpose of obtaining an upper bound, we may work with the new renewal process, and we will simply assume that the original renewal process $N_{0}$ has interarrival times that are bounded from below by a positive constant.

We observe that $I^{*}(0)$ is an infinitely divisible random variable with Lévy measure given by

$$
\mu(B)=\lambda_{0} \int_{0}^{\infty} \mathrm{P}\left(A^{(c)}(x) \in B\right) \mathrm{d} x \quad \text { for } B \in \mathcal{B}(\mathbb{R}),
$$

where $A^{(c)}(x)$ is the total amount of data in a session belonging to a single cluster, initiated at 0 , that does not finish by time $x>0$, i.e. $A^{(c)}(x)=\sum_{j=1}^{N_{c}(0, x]}\left[T_{j}+Z_{j}-x\right]_{+}$. To see this, write $I^{*}(0)$ with respect to a Poisson random measure and use, for example, Lemma 2.2(i) of Kallenberg (2002). Without loss of generality, let $\lambda_{0}=1$. We have, therefore, the decomposition

$$
\mu(z, \infty)=\int_{0}^{\infty} \mathrm{P}\left(A^{c}(x)>z\right) \mathrm{d} x \leq I_{4,0}+I_{4,1}+I_{4,2}+I_{4,3},
$$

where

$$
\begin{aligned}
& I_{4,0}=\int_{0}^{z} \mathrm{P}\left(A^{(c)}(x)>z\right) \mathrm{d} x, \\
& I_{4,1}=\int_{z}^{\infty} \mathrm{P}\left(Z_{N_{c}(0, x]}>z+\left(x-T_{N_{c}(0, x]}\right), T_{K}>z\right) \mathrm{d} x, \\
& I_{4,2}=\mathrm{E}\left(\mathbf{1}_{\left\{T_{K}>z\right\}} \int_{z}^{\infty} \mathrm{P}\left(\bigcup_{j=0}^{N_{c}(0, x]-1}\left\{Z_{j}>x-T_{j}\right\} \mid \mathcal{F}\right) \mathrm{d} x\right), \\
& I_{4,3}=\mathrm{E}\left(\mathbf{1}_{\left\{T_{K} \leq z\right\}} \int_{z}^{\infty} \mathrm{P}\left(\bigcup_{j=0}^{K}\left\{Z_{j}>x-T_{j}\right\} \mid \mathcal{F}\right) \mathrm{d} x\right),
\end{aligned}
$$

where $\mathcal{F}$ is the $\sigma$-field generated by the cluster point process $N_{c}$.

Let $z \geq 1$. Then Proposition 4.1 of Faÿ et al. (2006) gives

$$
I_{4,0} \leq z \mathrm{P}\left(\sum_{j=0}^{K} Z_{j}>z\right) \leq C_{0} z^{1-\alpha_{1}}
$$

Next,

$$
\begin{aligned}
I_{4,1} \leq & \mathrm{E}\left(\mathbf{1}_{\left\{T_{K}>z\right\}} \sum_{k=N_{0}(0, z]}^{K-1} \int_{T_{k}}^{T_{k+1}} \mathrm{P}\left(Z_{k}>z+\left(x-T_{k}\right) \mid \mathcal{F}\right) \mathrm{d} x\right) \\
& +\mathrm{E}\left(\mathbf{1}_{\left\{T_{K}>z\right\}} \int_{T_{K}}^{\infty} \mathrm{P}\left(Z_{K}>z+\left(x-T_{K}\right) \mid \mathcal{F}\right) \mathrm{d} x\right) .
\end{aligned}
$$


By Markov's inequality we obtain

$$
\begin{aligned}
I_{4,1} \leq & C_{1} \mathrm{E}\left(\mathbf{1}_{\left\{T_{K}>z\right\}} \sum_{k=N_{0}(0, z]}^{K-1} \int_{T_{k}}^{T_{k+1}}\left(z+\left(x-T_{k}\right)\right)^{-2} \mathrm{~d} x\right) \\
& +C_{2} \mathrm{E}\left(\mathbf{1}_{\left\{T_{K}>z\right\}} \int_{T_{K}}^{\infty}\left(z+\left(x-T_{K}\right)\right)^{-2} \mathrm{~d} x\right) \\
= & C_{1} \mathrm{E}\left(\mathbf{1}_{\left\{T_{K}>z\right\}} \sum_{k=N_{0}(0, z]}^{K-1}\left[z^{-1}-\left(z+X_{k+1}\right)^{-1}\right]\right)+C_{2} \mathrm{E}\left(\mathbf{1}_{\left\{T_{K}>z\right\}} z^{-1}\right) \\
\leq & C_{3} z^{-1} \mathrm{E}\left(\mathbf{1}_{\left\{T_{K}>z\right\}} K\right) .
\end{aligned}
$$

Note that

$$
\mathrm{E}\left(\mathbf{1}_{\left\{T_{K}>z\right\}} K\right)=\mathrm{E}(K) \sum_{k=1}^{\infty} \mathrm{P}(\tilde{K}=k) \mathrm{P}\left(X_{1}+\cdots+X_{k}>z\right)=\mathrm{E}(K) \mathrm{P}\left(T_{\tilde{K}}>z\right),
$$

where $\tilde{K}$ is a positive integer-valued random variable with $\mathrm{P}(\tilde{K}=k)=k \mathrm{P}(K=k) / \mathrm{E}(K)$, $k \in \mathbb{N}$. Furthermore, by Karamata's theorem,

$$
\mathrm{P}(\tilde{K}>n) \sim \frac{1}{\mathrm{E}(K)} \frac{\alpha}{\alpha-1} n \mathrm{P}(K>n) \quad \text { as } n \rightarrow \infty .
$$

Hence, by Proposition 4.1,

$$
\mathrm{E}\left(\mathbf{1}_{\left\{T_{K}>z\right\}} K\right) \sim C_{4} \bar{F}(z)^{-1} \mathrm{P}\left(K>\bar{F}(z)^{-1}\right) \leq C_{5} z^{-\left(\alpha_{1}-1\right) / \beta_{2}},
$$

and, thus,

$$
I_{4,1} \leq C_{6} z^{-1} z^{-\left(\alpha_{1}-1\right) / \beta_{2}} \leq C_{7} z^{-\left(\alpha_{1}-1\right) / \beta_{2}} .
$$

Next, we decompose $I_{4,2}$ into

$$
I_{4,2}=I_{4,2,1}+I_{4,2,2}
$$

where

$$
\begin{aligned}
& I_{4,2,1}=\mathrm{E}\left(\mathbf{1}_{\left\{T_{K}>z\right\}} \int_{z}^{T_{K+1}} \mathrm{P}\left(\bigcup_{j=0}^{N_{0}(0, x]-1}\left\{Z_{j}>x-T_{j}\right\} \mid \mathcal{F}\right) \mathrm{d} x\right) \\
& I_{4,2,2}=\mathrm{E}\left(\mathbf{1}_{\left\{T_{K}>z\right\}} \int_{T_{K+1}}^{\infty} \mathrm{P}\left(\bigcup_{j=0}^{K}\left\{Z_{j}>x-T_{K}\right\} \mid \mathcal{F}\right) \mathrm{d} x\right) .
\end{aligned}
$$

Then

$$
\begin{aligned}
I_{4,2,1} & =\mathrm{E}\left(\mathbf{1}_{\left\{T_{K}>z\right\}} \int_{z}^{T_{K+1}} \sum_{j=0}^{N_{0}(0, x]-1} \mathrm{P}\left(Z_{j}>x-T_{j} \mid \mathcal{F}\right) \mathrm{d} x\right) \\
& \leq \mathrm{E}\left(\mathbf{1}_{\left\{T_{K}>z\right\}} \sum_{k=N_{0}(0, z]}^{K} \int_{T_{k}}^{T_{k+1}} \sum_{j=0}^{k-1} \mathrm{P}\left(Z_{j}>x-T_{j} \mid \mathcal{F}\right) \mathrm{d} x\right) .
\end{aligned}
$$


Again, applying Markov's inequality and (5.13) leads to

$$
\begin{aligned}
I_{4,2,1} & \leq C_{8} \mathrm{E}\left(\mathbf{1}_{\left\{T_{K}>z\right\}} \sum_{k=N_{0}(0, z]}^{K} \sum_{j=0}^{k-1} \int_{T_{k}}^{T_{k+1}}\left(x-T_{j}\right)^{-2} \mathrm{~d} x\right) \\
& =C_{8} \mathrm{E}\left(\mathbf{1}_{\left\{T_{K}>z\right\}} \sum_{j=0}^{K-1}\left[\left(T_{N_{0}(0, z] \vee(j+1)}-T_{j}\right)^{-1}-\left(T_{K+1}-T_{j}\right)^{-1}\right]\right) \\
& \leq C_{9} \mathrm{E}\left(\mathbf{1}_{\left\{T_{K}>z\right\}} \sum_{j=1}^{K} X_{j}^{-1}\right) \\
& \leq C_{9} \delta^{-1} \mathrm{E}\left(\mathbf{1}_{\left\{T_{K}>z\right\}} K\right) \\
& \sim C_{10} z^{-\left(\alpha_{1}-1\right) / \beta_{2}} \text { as } z \rightarrow \infty .
\end{aligned}
$$

Furthermore, as above, by Markov's inequality and (5.13),

$$
I_{4,2,2} \leq \mathrm{E}\left(Z^{2}\right) \mathrm{E}\left(\mathbf{1}_{\left\{T_{K}>z\right\}} K \int_{T_{K}+1}^{\infty}\left(x-T_{K}\right)^{-2} \mathrm{~d} x\right) \leq C_{11} \mathrm{E}\left(\mathbf{1}_{\left\{T_{K}>z\right\}} K\right) \leq C_{12} z^{-\left(\alpha_{1}-1\right) / \beta_{2}}
$$

for large enough $z$. We decompose $I_{4,3}$ into

$$
\begin{aligned}
I_{4,3}= & \mathrm{E}\left(\mathbf{1}_{\left\{z / 2 \leq T_{K} \leq z\right\}} \int_{z}^{z+1} \mathrm{P}\left(\bigcup_{j=0}^{K}\left\{Z_{j}>x-T_{j}\right\} \mid \mathcal{F}\right) \mathrm{d} x\right) \\
& +\mathrm{E}\left(\mathbf{1}_{\left\{z / 2 \leq T_{K} \leq z\right\}} \int_{z+1}^{\infty} \mathrm{P}\left(\bigcup_{j=0}^{K}\left\{Z_{j}>x-T_{j}\right\} \mid \mathcal{F}\right) \mathrm{d} x\right) \\
& +\mathrm{E}\left(\mathbf{1}_{\left\{T_{K}<z / 2\right\}} \int_{z}^{\infty} \mathrm{P}\left(\bigcup_{j=0}^{K}\left\{Z_{j}>x-T_{j}\right\} \mid \mathcal{F}\right) \mathrm{d} x\right) \\
= & : I_{4,3,1}+I_{4,3,2}+I_{4,3,3} .
\end{aligned}
$$

On the one hand, by Proposition 4.1 of Faÿ et al. (2006),

$$
I_{4,3,1} \leq \mathrm{P}\left(T_{K}>\frac{z}{2}\right) \leq C_{13} z^{-1 / \beta_{2}} .
$$

On the other hand, by Markov's inequality and (5.13), we obtain

$$
I_{4,3,2} \leq C_{14} \mathrm{E}\left(\mathbf{1}_{\left\{z / 2 \leq T_{K} \leq z\right\}} K \int_{z+1}^{\infty}\left(x-T_{K}\right)^{-2} \mathrm{~d} x\right) \leq C_{15} \mathrm{E}\left(\mathbf{1}_{\left\{T_{K}>z / 2\right\}} K\right) \leq C_{16} z^{-\left(\alpha_{1}-1\right) / \beta_{2}} .
$$

Finally, another application of Markov's inequality gives

$$
I_{4,3,3} \leq \mathrm{E}\left(\mathbf{1}_{\left\{T_{K}<z / 2\right\}} K \int_{z}^{\infty}\left(x-T_{K}\right)^{-2} \mathrm{~d} x\right) \leq C_{17} \mathrm{E}(K) z^{-1} .
$$

A conclusion of (5.10)-(5.21) is

$$
\mu(z, \infty) \leq C_{18} z^{1-\alpha_{1}}+C_{19} z^{-\left(\alpha_{1}-1\right) / \beta_{2}}+C_{20} z^{-1 / \beta_{2}}+C_{21} z^{-1} \leq C_{22} z^{-\left(\alpha_{1}-1\right) / \beta_{2}} .
$$

Hence, a stochastic domination argument and the fact that the tail of a regularly varying Lévy measure is equivalent to the tail of its distribution function give the result. 


\section{Auxiliary results}

A number of lemmas and other auxiliary results are collected in this section. We start with a lemma that clarifies the behavior of the normalizing sequence $\left(b_{n}\right)$ in Theorem 3.1.

Lemma 6.1. Let Assumption 2.1 hold, and let $\left(b_{n}\right)$ be defined as in (3.1). Then

$$
\begin{gathered}
\lim _{n \rightarrow \infty}\left(\bar{F}\left(M_{n}\right) b_{n}\right)^{-1}=0, \\
\lim _{n \rightarrow \infty} n M_{n} b_{n}^{-2}=0 .
\end{gathered}
$$

Proof. For large $n$, we have, by Potter's theorem,

$$
\bar{F}\left(M_{n}\right) b_{n}=n^{1 / 2} M_{n}^{1 / 2} \mathrm{P}\left(K>\bar{F}\left(M_{n}\right)\right)^{-1 / 2} \geq n^{1 / 2} M_{n}^{1 / 2} \bar{F}\left(M_{n}\right)^{\alpha_{2} / 2} \geq n^{1 / 2} M_{n}^{1 / 2} M_{n}^{-\alpha_{2} / 2 \beta_{1}} .
$$

Since $\left(\beta_{1}-\alpha_{2}\right) /\left(2 \beta_{1}\right)>0$ and $M_{n} \rightarrow \infty$ as $n \rightarrow \infty$, we obtain

$$
\left(\bar{F}\left(M_{n}\right) b_{n}\right)^{-1} \leq n^{-1 / 2} M_{n}^{-\left(\beta_{1}-\alpha_{2}\right) / \beta_{1}} \rightarrow 0 \quad \text { as } n \rightarrow \infty .
$$

Finally, (6.2) follows from

$$
n M_{n} b_{n}^{-2}=\bar{F}\left(M_{n}\right)^{2} \mathrm{P}\left(K>\bar{F}\left(M_{n}\right)^{-1}\right)^{-1} \leq \bar{F}\left(M_{n}\right)^{2-\alpha_{2}} \rightarrow 0 \quad \text { as } n \rightarrow \infty .
$$

The next result is a simple consequence of the strong Markov property which is useful in various places in our arguments.

Lemma 6.2. Let $f$ and $g$ be measurable functions, and let $f$ be increasing. Suppose that $N_{0}$ is a renewal process. Then, for $w, \delta>0$,

$$
\begin{aligned}
\mathrm{E}(f & \left.\left(N_{0}(w, w+\delta]\right) g\left(N_{0}(0, w]\right) \mathbf{1}_{\left\{N_{0}(0, w] \neq N_{0}(0, w+\delta]\right\}}\right) \\
& \leq \mathrm{E}\left(f\left(1+N_{0}(0, \delta]\right)\right) \mathrm{E}\left(g\left(N_{0}(0, w]\right) \mathbf{1}_{\left\{N_{0}(0, w] \neq N_{0}(0, w+\delta]\right\}}\right) .
\end{aligned}
$$

Proof. Condition on the time and the number of first arrivals after $w$, and use the i.i.d. assumption of the interarrival times.

The next lemma gives a simple estimate on the probability of having 'too many' arrivals within a time interval.

Lemma 6.3. Let $\left(X_{k}\right)$ be an i.i.d. sequence of positive random variables with distribution function $F$, such that $\bar{F} \in \mathcal{R}_{-1 / \beta}, 0<1 / \beta<1$, and let $h$ be the generalized tail inverse function (2.2). Let $T_{m}=\sum_{k=1}^{m} X_{k}, m \in \mathbb{N}$. For any $\delta>0$ such that $\bar{F}(\delta)>0$ and $m \geq 1$,

(i) we have

$$
\mathrm{P}\left(T_{m} \leq \delta\right) \leq F(\delta)^{m} \leq \mathrm{e}^{-m \bar{F}(\delta)}
$$

(ii) if $x \geq \delta / h(m)$ then, for any $\beta_{1}<\beta<\beta_{2}$, we have

$$
\mathrm{P}\left(T_{m} \leq h(m) x\right) \leq \exp \left(-C \min \left(x^{-1 / \beta_{1}}, x^{-1 / \beta_{2}}\right)\right)
$$

for some $C=C\left(\delta, \beta_{1}, \beta_{2}\right)$. 
Proof. Trivially, for $\delta>0$,

$$
\mathrm{P}\left(T_{m} \leq \delta\right) \leq[1-\bar{F}(\delta)]^{m} .
$$

Now (6.3) follows from the fact that $\left(1-a^{-1}\right)^{a} \leq \mathrm{e}^{-1}$ for $a \geq 1$ and Potter's bounds (cf. Resnick (2006, p. 36)) give (6.4).

The following simple result on convolution tails of random variables with infinite mean is often useful.

Lemma 6.4. Let $\left(X_{k}\right)$ be an i.i.d. sequence of positive random variables with distribution function $F$, such that $\bar{F} \in \mathcal{R}_{-1 / \beta}, 0<1 / \beta<1$. Then there exist $K>0$ and $n_{0} \in \mathbb{N}$ such that, for any $x>0$ and $n \geq n_{0}$,

$$
\overline{F^{n^{*}}}(x) \leq K n \bar{F}(x) .
$$

Proof. Suppose that the statement is not true. Then, for each $j \geq 1$, there exist an $n_{j} \geq j$ and an $x_{j}>0$ such that

$$
\overline{F^{n_{j} *}}\left(x_{j}\right) \geq j n_{j} \bar{F}\left(x_{j}\right) .
$$

Let $h$ be the generalized tail inverse function (2.2). Assume first that there is a sequence $j_{k} \uparrow \infty$ as $k \rightarrow \infty$ such that

$$
\lim _{k \rightarrow \infty} \frac{x_{j_{k}}}{h\left(n_{j_{k}}\right)}=\infty
$$

This implies that $\lim _{k \rightarrow \infty} n_{j_{k}} \bar{F}\left(x_{j_{k}}\right)=0$. Therefore, by Theorem 9.1 of Denisov et al. (2008) we obtain

$$
\lim _{k \rightarrow \infty}\left|\frac{\overline{F^{n_{j_{k}} *}}\left(x_{j_{k}}\right)}{n_{j_{k}} \bar{F}\left(x_{j_{k}}\right)}-1\right|=0
$$

which contradicts (6.5).

Next, we suppose that there exists an $M>0$ such that

$$
x_{j} \leq M h\left(n_{j}\right) \quad \text { for all } j \in \mathbb{N} .
$$

Then

$$
n_{j} \bar{F}\left(x_{j}\right) \geq n_{j} \bar{F}\left(M h\left(n_{j}\right)\right) \rightarrow M^{-1 / \beta} \quad \text { as } j \rightarrow \infty
$$

by the regular variation of $\bar{F}$. Thus, (6.5) results in

$$
\overline{F^{n_{j} *}}\left(x_{j}\right) \geq j n_{j} \bar{F}\left(x_{j}\right) \rightarrow \infty \quad \text { as } j \rightarrow \infty .
$$

Since $\overline{F^{n_{j *}}}$ is bounded by 1 , this is impossible. Hence, the claim follows.

\subsection{Auxiliary results for the proof of Lemma 5.1}

The next series of lemmas provides estimates needed to prove the convergence of $\xi_{n}^{+}$in Lemma 5.1. We continue to use the same notation.

Lemma 6.5. Let Assumption 2.1 hold, and let $I_{1,1}(n)$ be as in (5.4). Then

$$
\lim _{n \rightarrow \infty} I_{1,1}(n)=\frac{2}{2+\beta-\alpha} t^{(2+\beta-\alpha) / \beta} \int_{0}^{\infty} y^{-(2+\beta-\alpha) / \beta} \mathrm{P}\left(S_{1 / \beta}(1) \leq y\right) \mathrm{d} y .
$$


Proof. We have, by the independence of $K$ and $N_{0}$,

$$
\begin{aligned}
\mathrm{E}\left(N_{c}[0, u]^{2} \mathbf{1}_{\left\{K+1 \leq \varepsilon b_{n}\right\}}\right) & =\int_{0}^{\varepsilon^{2} b_{n}^{2}} \mathrm{P}\left(\left(N_{0}[0, u]^{2} \wedge(K+1)^{2}\right) \mathbf{1}_{\left\{K+1 \leq \varepsilon b_{n}\right\}}>x\right) \mathrm{d} x \\
& =2 \int_{0}^{\varepsilon b_{n}} y \mathrm{P}\left(N_{0}[0, u]>y\right) \mathrm{P}\left(y<K+1 \leq \varepsilon b_{n}\right) \mathrm{d} y
\end{aligned}
$$

Hence,

$$
\begin{aligned}
I_{1,1}(n)= & 2 \frac{n}{b_{n}^{2}} \int_{0}^{M_{n} t} \int_{0}^{\varepsilon b_{n}} y \mathrm{P}\left(N_{0}[0, u]>y\right) \mathrm{P}\left(y<K+1 \leq \varepsilon b_{n}\right) \mathrm{d} y \mathrm{~d} u \\
= & 2 \frac{n}{b_{n}^{2}} \int_{0}^{\varepsilon b_{n}} y \mathrm{P}\left(y<K+1 \leq \varepsilon b_{n}\right) \int_{0}^{M_{n} t} \mathrm{P}\left(T_{\lfloor y\rfloor} \leq u\right) \mathrm{d} u \mathrm{~d} y \\
= & 2 \frac{n}{b_{n}^{2}} \int_{0}^{\varepsilon b_{n}} y \mathrm{P}\left(y<K+1 \leq \varepsilon b_{n}\right) \mathrm{E}\left(M_{n} t-T_{\lfloor y\rfloor}\right)_{+} \mathrm{d} y \\
= & 2 \frac{n}{b_{n}^{2}} \bar{F}\left(M_{n}\right)^{-2} \\
& \times \int_{0}^{\varepsilon b_{n} \bar{F}\left(M_{n}\right)} z \mathrm{P}\left(z \bar{F}\left(M_{n}\right)^{-1}<K+1 \leq \varepsilon b_{n}\right) \mathrm{E}\left(M_{n} t-T_{\left\lfloor z \bar{F}\left(M_{n}\right)^{-1}\right\rfloor}\right)_{+} \mathrm{d} z \\
= & \left.2 \int_{0}^{\varepsilon} z \frac{T_{\left\lfloor z \bar{F}\left(M_{n}\right)^{-1}\right\rfloor}}{M_{n}}\right)_{+} \mathrm{d} z \\
& +2 \int_{\varepsilon}^{\varepsilon b_{n} \bar{F}\left(M_{n}\right)} \mathrm{P}(K)^{-1}<\bar{P}\left(z \bar{F}\left(M_{n}\right)^{-1}<K+1 \leq \varepsilon b_{n}\right) \\
= & J_{1}(n, \varepsilon)+J_{2}\left(t-\frac{T_{\left\lfloor z \bar{F}\left(M_{n}\right)^{-1}\right\rfloor}}{M_{n}}\right)_{+} \mathrm{d} z \\
& \mathrm{P}\left(K>\bar{F}\left(M_{n}\right)^{-1}\right)
\end{aligned}
$$

By Karamata's theorem,

$$
\begin{aligned}
J_{1}(n, \varepsilon) \leq & \frac{2 t}{\mathrm{P}\left(K>\bar{F}\left(M_{n}\right)^{-1}\right)} \int_{0}^{\varepsilon} z \mathrm{P}\left(K+1>z \bar{F}\left(M_{n}\right)^{-1}\right) \mathrm{d} z \\
= & \frac{\mathrm{P}\left(K+1>\bar{F}\left(M_{n}\right)^{-1}\right)}{\mathrm{P}\left(K>\bar{F}\left(M_{n}\right)^{-1}\right)} \frac{2 t}{\bar{F}\left(M_{n}\right)^{-2} \mathrm{P}\left(K+1>\bar{F}\left(M_{n}\right)^{-1}\right)} \\
& \times \int_{0}^{\varepsilon \bar{F}\left(M_{n}\right)^{-1}} z \mathrm{P}(K+1>z) \mathrm{d} z \\
\rightarrow & \frac{2 t \alpha}{2-\alpha} \varepsilon^{2-\alpha} \quad \text { as } n \rightarrow \infty,
\end{aligned}
$$

and we conclude that $\lim _{\varepsilon \downarrow 0} \lim _{n \rightarrow \infty} J_{1}(n, \varepsilon)=0$. We estimate $J_{2}(n, \varepsilon)$ as follows. By Potter's inequality, there exists a $C_{1}>0$ such that, for $z \geq \varepsilon$ and large $n$,

$$
\frac{\mathrm{P}\left(K+1>z \bar{F}\left(M_{n}\right)^{-1}\right)}{\mathrm{P}\left(K>\bar{F}\left(M_{n}\right)^{-1}\right)} \leq C_{1} z^{-\alpha_{1}} .
$$

Similarly, Potter's inequality leads to

$$
\frac{h\left(\left\lfloor z \bar{F}\left(M_{n}\right)^{-1}\right\rfloor\right)}{M_{n}} \geq \frac{h\left(z \bar{F}\left(M_{n}\right)^{-1}-1\right)}{h\left(\bar{F}\left(M_{n}\right)^{-1}+1\right)} \geq C_{2} z^{\beta_{1}} \quad \text { for } z \geq \varepsilon .
$$


If we define $m_{n}=\left\lfloor z \bar{F}\left(M_{n}\right)^{-1}\right\rfloor$ then, for $\delta>0$ such that $F(\delta)<1$,

$$
\begin{aligned}
& \mathrm{E}\left(t-\frac{T_{\left\lfloor z \bar{F}\left(M_{n}\right)^{-1}\right\rfloor}}{M_{n}}\right)_{+}=\mathrm{E}\left(t-\frac{T_{m_{n}}}{h\left(m_{n}\right)} \frac{h\left(m_{n}\right)}{M_{n}}\right)_{+} \\
& \leq \mathrm{E}\left(t-\frac{T_{m_{n}}}{h\left(m_{n}\right)} C_{2} z^{\beta_{1}}\right)_{+} \\
& =C_{2} z^{\beta_{1}}\left[\int_{0}^{\delta / h\left(m_{n}\right)}+\int_{\delta / h\left(m_{n}\right)}^{C_{2}^{-1} t z^{-\beta_{1}}}\right] \mathrm{P}\left(\frac{T_{m_{n}}}{h\left(m_{n}\right)} \leq x\right) \mathrm{d} x \\
& =: C_{2} z^{\beta_{1}}\left[V_{1}(n, z)+V_{2}(n, z)\right] \text {. }
\end{aligned}
$$

We have, by (6.3), for large $n$,

$$
V_{1}(n, z) \leq \frac{\delta}{h\left(m_{n}\right)} \mathrm{P}\left(T_{m_{n}} \leq \delta\right) \leq \delta \mathrm{e}^{-m_{n} \bar{F}(\delta)} \leq C_{3}^{-1} \exp \left(-C_{3} z\right) \quad \text { for some } C_{3}>0,
$$

since $m_{n} \geq z$ for large $n$. Furthermore, by (6.4),

$$
\begin{aligned}
V_{2}(n, z) & \leq \int_{0}^{C_{2}^{-1} t z^{-\beta_{1}}} \exp \left(-C_{4} \min \left(x^{-1 / \beta_{1}}, x^{-1 / \beta_{2}}\right)\right) \mathrm{d} x \\
& \leq C_{5}^{-1} t z^{-\beta_{1}} \exp \left(-C_{5} z\right) \\
& \leq C_{6}^{-1} \exp \left(-C_{6} z\right)
\end{aligned}
$$

for some $C_{4}, C_{5}, C_{6}>0$. Hence, we have

$$
\mathrm{E}\left(t-\frac{T_{\left\lfloor z \bar{F}\left(M_{n}\right)^{-1}\right\rfloor}}{M_{n}}\right)_{+} \leq C_{2} z^{\beta_{1}}\left[V_{1}(n, z)+V_{2}(n, z)\right] \leq C_{7}^{-1} z^{\beta_{1}} \exp \left(-C_{7} z\right),
$$

and so by the dominated convergence theorem, (2.3), and the regular variation of $\bar{F}_{K}$,

$$
\lim _{n \rightarrow \infty} J_{2}(n, \varepsilon)=2 \int_{\varepsilon}^{\infty} z^{1-\alpha} \mathrm{E}\left(t-z^{\beta} S_{1 / \beta}(1)\right)_{+} \mathrm{d} z .
$$

Therefore, by (6.6),

$$
\begin{aligned}
\lim _{n \rightarrow \infty} I_{1,1}(n) & =2 \int_{0}^{\infty} z^{1-\alpha} \mathrm{E}\left(t-z^{\beta} S_{1 / \beta}(1)\right)_{+} \mathrm{d} z \\
& =\frac{2}{\beta} t^{(2+\beta-\alpha) / \beta} \int_{0}^{\infty} x^{-(2+\beta-\alpha) / \beta} \mathrm{E}\left(1-x^{-1} S_{1 / \beta}(1)\right)_{+} \mathrm{d} x \\
& =\frac{2}{\beta} t^{(2+\beta-\alpha) / \beta} \int_{0}^{\infty} x^{-(2+\beta-\alpha) / \beta-1} \int_{0}^{x} \mathrm{P}\left(S_{1 / \beta}(1) \leq z\right) \mathrm{d} z \mathrm{~d} x \\
& =\frac{2}{2+\beta-\alpha} t^{(2+\beta-\alpha) / \beta} \int_{0}^{\infty} z^{-(2+\beta-\alpha) / \beta} \mathrm{P}\left(S_{1 / \beta}(1) \leq z\right) \mathrm{d} z
\end{aligned}
$$

Lemma 6.6. Let Assumption 2.1 hold, and let $I_{1,2}(n)$ be as in (5.4). Then

$$
\lim _{n \rightarrow \infty} I_{1,2}(n)=0 \text {. }
$$


Proof. By the independence of $K$ and $N_{0}$ we have

$$
\begin{aligned}
I_{1,2}(n) & \leq \frac{n}{b_{n}^{2}} \mathrm{E}\left(\mathbf{1}_{\left\{K+1>\varepsilon b_{n}\right\}} \int_{0}^{M_{n} t} N_{0}[0, u]^{2} \mathrm{~d} u\right) \\
& =\frac{n}{b_{n}^{2}} \mathrm{P}\left(K+1>\varepsilon b_{n}\right) \int_{0}^{M_{n} t} \mathrm{E}\left(N_{0}[0, u]^{2}\right) \mathrm{d} u .
\end{aligned}
$$

Thus,

$$
\begin{aligned}
I_{1,2}(n) & \leq \frac{n}{b_{n}^{2}} \mathrm{P}\left(K+1>\varepsilon b_{n}\right) \int_{0}^{M_{n} t} \int_{0}^{\infty} \mathrm{P}\left(N_{0}[0, u]^{2}>x\right) \mathrm{d} x \mathrm{~d} u \\
& \leq 2 \frac{n}{b_{n}^{2}} M_{n} t \mathrm{P}\left(K+1>\varepsilon b_{n}\right) \int_{0}^{\infty} z \mathrm{P}\left(T_{\lfloor z\rfloor} \leq M_{n} t\right) \mathrm{d} z .
\end{aligned}
$$

By (6.3), Potter's inequality, and (6.1),

$$
\begin{aligned}
I_{1,2}(n) & \leq C_{1} \frac{n}{b_{n}^{2}} M_{n} t \mathrm{P}\left(K+1>\varepsilon b_{n}\right) \int_{0}^{\infty} z \exp \left(-\lfloor z\rfloor \bar{F}\left(M_{n} t\right)\right) \mathrm{d} z \\
& \leq C_{2} \frac{n}{b_{n}^{2}} M_{n} t \mathrm{P}\left(K+1>\varepsilon b_{n}\right) \bar{F}\left(M_{n} t\right)^{-2} \\
& =C_{3} t^{1-\alpha_{1}} \frac{\mathrm{P}\left(K>\varepsilon b_{n}-1\right)}{\mathrm{P}\left(K>\bar{F}\left(M_{n}\right)^{-1}\right)} \rightarrow 0 \quad \text { as } n \rightarrow \infty .
\end{aligned}
$$

Lemma 6.7. Let Assumption 2.1 hold, and let $I_{2,1}(n)$ be as in (5.5). Then

$$
\lim _{n \rightarrow \infty} I_{2,1}(n)=0 .
$$

Proof. Suppose that $\varepsilon=1$. The independence of $K$ and $N_{0}$ results in

$$
\begin{aligned}
I_{2,1}(n) & =n \mathrm{P}\left(K+1>b_{n}\right) \int_{0}^{M_{n} t} \mathrm{P}\left(N_{0}[0, u]>b_{n}\right) \mathrm{d} u \\
& =n \mathrm{P}\left(K+1>b_{n}\right) \int_{0}^{M_{n} t} \mathrm{P}\left(T_{\left\lfloor b_{n}\right\rfloor} \leq u\right) \mathrm{d} u .
\end{aligned}
$$

As in (6.3), we obtain

$$
\begin{aligned}
I_{2,1}(n) & \leq n \mathrm{P}\left(K+1>b_{n}\right) \int_{0}^{M_{n}} \exp \left(-\left\lfloor b_{n}\right\rfloor \bar{F}(u)\right) \mathrm{d} u \\
& \leq n \mathrm{P}\left(K+1>b_{n}\right) M_{n} \exp \left(-\left(b_{n}-1\right) \bar{F}\left(M_{n}\right)\right) \\
& =\frac{\mathrm{P}\left(K+1>b_{n}\right)}{\mathrm{P}\left(K>\bar{F}\left(M_{n}\right)^{-1}\right)}\left(\bar{F}\left(M_{n}\right) b_{n}\right)^{2} \exp \left(-\left(b_{n}-1\right) \bar{F}\left(M_{n}\right)\right) \\
& \rightarrow 0 \text { as } n \rightarrow \infty,
\end{aligned}
$$

since $b_{n} \bar{F}\left(M_{n}\right) \rightarrow \infty$ as $n \rightarrow \infty$ by Lemma 6.1 .

Lemma 6.8. Let Assumption 2.1 hold, and let $I_{2,2}(n)$ be as in (5.5). Then

$$
\lim _{n \rightarrow \infty} I_{2,2}(n)=0 \text {. }
$$


Proof. Suppose that $\varepsilon=1$ and $t=1$. Then

$$
\begin{aligned}
I_{2,2}(n) & =n \int_{1}^{\infty} \mathrm{P}\left(K+1>b_{n} x\right) \int_{0}^{M_{n}} \mathrm{P}\left(N_{0}[0, u]>b_{n} x\right) \mathrm{d} u \mathrm{~d} x \\
& =n \int_{1}^{\infty} \mathrm{P}\left(K+1>b_{n} x\right) \int_{0}^{M_{n}} \mathrm{P}\left(T_{\left.\left\lfloor b_{n} x\right\rfloor \leq u\right) \mathrm{d} u \mathrm{~d} x .}\right.
\end{aligned}
$$

Again, as in (6.3), we obtain, by (6.1),

$$
\begin{aligned}
I_{2,2}(n) & \leq n \int_{1}^{\infty} \mathrm{P}\left(K+1>b_{n} x\right) \int_{0}^{M_{n}} \exp \left(-\left\lfloor b_{n} x\right\rfloor \bar{F}(u)\right) \mathrm{d} u \mathrm{~d} x \\
& \leq n M_{n} \int_{1}^{\infty} \mathrm{P}\left(K+1>b_{n} x\right) \exp \left(-\left(b_{n} x-1\right) \bar{F}\left(M_{n}\right)\right) \mathrm{d} x \\
& \leq \mathrm{e}^{1} n M_{n} \int_{1}^{\infty} \exp \left(-b_{n} x \bar{F}\left(M_{n}\right)\right) \mathrm{d} x \\
& \leq \mathrm{e}^{1} n M_{n}\left(b_{n} \bar{F}\left(M_{n}\right)\right)^{-1} \exp \left(-b_{n} \bar{F}\left(M_{n}\right)\right) \\
& \leq \mathrm{e}^{1}\left(n^{1 / 2} M_{n}^{H-1 / \beta}\right)^{\max \{2,1 /(H-1 / \beta)\}}\left(b_{n} \bar{F}\left(M_{n}\right)\right)^{-1} \exp \left(-b_{n} \bar{F}\left(M_{n}\right)\right) \\
& \leq C_{1}\left(b_{n} \bar{F}\left(M_{n}\right)\right)^{\max \{2,1 /(H-1 / \beta)\}-1} \exp \left(-b_{n} \bar{F}\left(M_{n}\right)\right) \\
& \rightarrow 0 \quad \text { as } n \rightarrow \infty
\end{aligned}
$$

which is the result.

\subsection{Auxiliary results for the proof of Lemma 5.2}

The next several results deal with the convergence of $\xi_{n}^{-}$in Lemma 5.2.

Lemma 6.9. Let Assumption 2.1 hold, and let $H_{n}^{(1)}(w)$ be as in (5.8). Then

$$
\lim _{n \rightarrow \infty} \frac{n M_{n}}{b_{n}^{2}} H_{n}^{(1)}(w)=\mathrm{E}\left((I(w+t)-I(w))^{2} I(w+t)^{-\alpha}\right) .
$$

Proof. We divide $H_{n}^{(1)}$ into three parts and define

$$
A_{n, w}=\left\{N_{0}\left(M_{n} w, M_{n}(w+t)\right] \leq \varepsilon b_{n}, K>N_{0}\left(0, M_{n}(w+t)\right]\right\} .
$$

For $M>0$, let

$$
\begin{aligned}
& H_{n}^{(1,1, M)}(w)=\mathrm{E}\left(N_{0}\left(M_{n} w, M_{n}(w+t)\right]^{2} \mathbf{1}_{\left\{M^{-1} \leq \bar{F}\left(M_{n}\right) N_{0}\left(0, M_{n}(w+t)\right] \leq M\right\}} \mathbf{1}_{A_{n, w}}\right), \\
& H_{n}^{(1,2, M)}(w)=\mathrm{E}\left(N_{0}\left(M_{n} w, M_{n}(w+t)\right]^{2} \mathbf{1}_{\left\{\bar{F}\left(M_{n}\right) N_{0}\left(0, M_{n}(w+t)\right]<M^{-1}\right\}} \mathbf{1}_{A_{n, w}}\right), \\
& H_{n}^{(1,3, M)}(w)=\mathrm{E}\left(N_{0}\left(M_{n} w, M_{n}(w+t)\right]^{2} \mathbf{1}_{\left\{\bar{F}\left(M_{n}\right) N_{0}\left(0, M_{n}(w+t)\right]>M\right\}} \mathbf{1}_{A_{n, w}}\right),
\end{aligned}
$$

so that

$$
H_{n}^{(1)}(w)=H_{n}^{(1,1, M)}(w)+H_{n}^{(1,2, M)}(w)+H_{n}^{(1,3, M)}(w) .
$$


Regularly varying functions converge uniformly on compact sets (cf. Bingham et al. (1987, Theorem 1.5.2)). Thus, (2.5) gives

$$
\begin{aligned}
& \frac{n M_{n}}{b_{n}^{2}} H_{n}^{(1,1, M)}(w) \\
& =\mathrm{E}\left(\frac{N_{0}\left(M_{n} w, M_{n}(w+t)\right]^{2}}{\bar{F}\left(M_{n}\right)^{-2}} \mathbf{1}_{\left\{N_{0}\left(M_{n} w, M_{n}(w+t)\right] \leq \varepsilon b_{n}, M^{-1} \leq \bar{F}\left(M_{n}\right) N_{0}\left(0, M_{n}(w+t)\right] \leq M\right\}}\right. \\
& \left.\quad \times \frac{\mathrm{P}\left(K>N_{0}\left(0, M_{n}(w+t)\right] \mid \mathcal{F}_{0}\right)}{\mathrm{P}\left(K>\bar{F}\left(M_{n}\right)^{-1}\right)}\right) \\
& \quad \mathrm{E}\left((I(w+t)-I(w))^{2} \mathbf{1}_{\left\{M^{-1} \leq I(w+t) \leq M\right\}} I(w+t)^{-\alpha}\right) \quad \text { as } n \rightarrow \infty,
\end{aligned}
$$

where $\mathcal{F}_{0}=\sigma\left(N_{0}\right)$. For the second summand of $H_{n}^{(1)}$, we have, for large $n$, by Potter's inequality,

$$
\begin{aligned}
& \frac{n M_{n}}{b_{n}^{2}} H_{n}^{(1,2, M)}(w) \\
& \leq \mathrm{E}\left(\left(\frac{N_{0}\left(M_{n} w, M_{n}(w+t)\right]}{\bar{F}\left(M_{n}\right)^{-1}}\right)^{2}\right. \\
& \left.\quad \quad \times \frac{\mathbf{1}_{\left\{K>N_{0}\left(0, M_{n}(w+t)\right]\right\}}}{\mathrm{P}\left(K>\bar{F}\left(M_{n}\right)^{-1}\right)} \mathbf{1}_{\left\{N_{0}\left(0, M_{n} w\right] \neq N_{0}\left(0, M_{n}(w+t)\right]<\bar{F}\left(M_{n}\right)^{-1} M^{-1}\right\}}\right) \\
& \leq C_{1} \mathrm{E}\left(\left(\frac{N_{0}\left(0, M_{n}(w+t)\right]}{\bar{F}\left(M_{n}\right)^{-1}}\right)^{2-\alpha_{2}} \mathbf{1}_{\left\{N_{0}\left(0, M_{n} w\right] \neq N_{0}\left(0, M_{n}(w+t)\right]<\bar{F}\left(M_{n}\right)^{-1} M^{-1}\right\}}\right) \\
& \leq C_{1} M^{\alpha_{2}-2} \\
& \rightarrow 0 \quad \text { as } M \rightarrow \infty
\end{aligned}
$$

By Potter's inequality, the last term of $H_{n}^{(1)}$ has the upper bound

$$
\begin{aligned}
& \frac{n M_{n}}{b_{n}^{2}} H_{n}^{(1,3, M)}(w) \\
& \leq \frac{n M_{n}}{b_{n}^{2}} \mathrm{E}\left(N_{0}\left(0, M_{n}(w+t)\right]^{2} \mathbf{1}_{\left\{\bar{F}\left(M_{n}\right) N_{0}\left(0, M_{n}(w+t)\right] \geq M\right\}} \mathrm{P}\left(K>N_{0}\left(0, M_{n}(w+t)\right] \mid \mathcal{F}_{0}\right)\right) \\
& \leq C_{2} \mathrm{E}\left(\left(\frac{N_{0}\left(0, M_{n}(w+t)\right]}{\bar{F}\left(M_{n}\right)^{-1}}\right)^{2-\alpha_{1}} \mathbf{1}_{\left\{N_{0}\left(0, M_{n}(w+t)\right] \geq \bar{F}\left(M_{n}\right)^{-1} M\right\}}\right) \\
& =C_{2} \int_{M^{2-\alpha_{1}}}^{\infty} \mathrm{P}\left(\left(\frac{N_{0}\left(0, M_{n}(w+t)\right]}{\bar{F}\left(M_{n}\right)^{-1}}\right)^{2-\alpha_{1}}>y\right) \mathrm{d} y \\
& \quad+C_{2} M^{2-\alpha_{1}} \mathrm{P}\left(\left(\frac{N_{0}\left(0, M_{n}(w+t)\right]}{\bar{F}\left(M_{n}\right)^{-1}}\right)^{2-\alpha_{1}}>M^{2-\alpha_{1}}\right) .
\end{aligned}
$$

The first term on the right-hand side above can be bounded as follows. For some constant 
$C_{3}>0$, we obtain, as in (6.3),

$$
\begin{aligned}
\int_{M^{2-\alpha_{1}}}^{\infty} & \mathrm{P}\left(\left(\frac{N_{0}\left(0, M_{n}(w+t)\right]}{\bar{F}\left(M_{n}\right)^{-1}}\right)^{2-\alpha_{1}}>y\right) \mathrm{d} y \\
\quad= & \left(2-\alpha_{1}\right) \int_{M}^{\infty} z^{1-\alpha_{1}} \mathrm{P}\left(N_{0}\left(0, M_{n}(w+t)\right]>z \bar{F}\left(M_{n}\right)^{-1}\right) \mathrm{d} z \\
\quad \leq & \left(2-\alpha_{1}\right) \int_{M}^{\infty} z^{1-\alpha_{1}} \mathrm{P}\left(T_{\left\lfloor z \bar{F}\left(M_{n}\right)^{-1}\right\rfloor+1} \leq M_{n}(w+t)\right) \mathrm{d} z \\
\quad \leq & \left(2-\alpha_{1}\right) \int_{M}^{\infty} z^{1-\alpha_{1}} \exp \left(-z \bar{F}\left(M_{n}\right)^{-1} \bar{F}\left(M_{n}(w+t)\right)\right) \mathrm{d} z \\
\quad \leq & C_{3}^{-1} \int_{M}^{\infty} z^{1-\alpha_{1}} \exp \left(-C_{3}(\omega+t)^{-1 / \beta_{1}} z\right) \mathrm{d} z \\
\quad \rightarrow & 0 \quad \text { as } M \rightarrow \infty .
\end{aligned}
$$

Similarly,

$$
M^{2-\alpha_{1}} \mathrm{P}\left(\left(\frac{N_{0}\left(0, M_{n}(w+t)\right]}{\bar{F}\left(M_{n}\right)^{-1}}\right)^{2-\alpha_{1}}>M^{2-\alpha_{1}}\right) \rightarrow 0 \quad \text { as } M \rightarrow \infty .
$$

Hence, the result follows.

Lemma 6.10. Let Assumption 2.1 hold, and let $H_{n}^{(2)}(w)$ be as in (5.8). Then

$$
\begin{aligned}
\lim _{n \rightarrow \infty} \frac{n M_{n}}{b_{n}^{2}} H_{n}^{(2)}(w)= & \mathrm{E}\left(\frac{\alpha}{2-\alpha} I(w+t)^{2-\alpha}+\frac{2 \alpha}{\alpha-1} I(w) I(w+t)^{1-\alpha}\right) \\
& -\mathrm{E}\left(I(w)^{2} I(w+t)^{-\alpha}+\frac{2}{(2-\alpha)(\alpha-1)} I(w)^{2-\alpha}\right) .
\end{aligned}
$$

Proof. We define

$$
\begin{aligned}
A_{n, M}=\left\{M^{-1} \leq \bar{F}\left(M_{n}\right) N_{0}\left(0, M_{n} w\right] \leq \bar{F}\left(M_{n}\right) N_{0}\left(0, M_{n}(w+t)\right] \leq M\right. & \\
& \left.N_{0}\left(M_{n} w, M_{n}(w+t)\right] \leq \varepsilon b_{n}\right\}
\end{aligned}
$$

and

$$
A_{M}=\left\{M^{-1} \leq I(w) \leq I(w+t) \leq M\right\}
$$

By Karamata's theorem and the uniform convergence of regularly varying functions on compact sets, we have

$$
\begin{aligned}
& \mathrm{E}\left(K^{2} \frac{\mathbf{1}_{\left\{K \leq N_{0}\left(0, M_{n}(w+t)\right]\right\}}-\mathbf{1}_{\left\{K \leq N_{0}\left(0, M_{n} w\right]\right\}}}{\bar{F}\left(M_{n}\right)^{-2} \mathrm{P}\left(K>\bar{F}\left(M_{n}\right)^{-1}\right)} \mathbf{1}_{A_{n, M}}\right) \\
& \quad \rightarrow \frac{\alpha}{2-\alpha} \mathrm{E}\left(\left(I(w+t)^{2-\alpha}-I(w)^{2-\alpha}\right) \mathbf{1}_{A_{M}}\right) \quad \text { as } n \rightarrow \infty
\end{aligned}
$$

and

$$
\begin{aligned}
& \mathrm{E}\left(\frac{N_{0}\left(0, M_{n} w\right]}{\bar{F}\left(M_{n}\right)^{-1}} K \frac{\mathbf{1}_{\left\{K>N_{0}\left(0, M_{n} w\right]\right\}}-\mathbf{1}_{\left\{K>N_{0}\left(0, M_{n}(w+t)\right]\right\}}}{\bar{F}\left(M_{n}\right)^{-1} \mathrm{P}\left(K>\bar{F}\left(M_{n}\right)^{-1}\right)} \mathbf{1}_{A_{n, M}}\right) \\
& \quad \rightarrow \frac{\alpha}{\alpha-1} \mathrm{E}\left(I(w)\left(I(w)^{1-\alpha}-I(w+t)^{1-\alpha}\right) \mathbf{1}_{A_{M}}\right) \quad \text { as } n \rightarrow \infty
\end{aligned}
$$


Furthermore,

$$
\begin{gathered}
\mathrm{E}\left(\frac{N_{0}\left(0, M_{n} w\right]^{2}}{\bar{F}\left(M_{n}\right)^{-2}} \frac{\mathbf{1}_{\left\{K>N_{0}\left(0, M_{n} w\right]\right\}}-\mathbf{1}_{\left\{K>N_{0}\left(0, M_{n}(w+t)\right]\right\}}}{\mathrm{P}\left(K>\bar{F}\left(M_{n}\right)^{-1}\right)} \mathbf{1}_{A_{n, M}}\right) \\
\rightarrow \mathrm{E}\left(I(w)^{2}\left(I(w)^{-\alpha}-I(w+t)^{-\alpha}\right) \mathbf{1}_{A_{M}}\right) \quad \text { as } n \rightarrow \infty .
\end{gathered}
$$

Thus, (6.7)-(6.9) give us

$$
\begin{aligned}
\lim _{M \rightarrow \infty} & \lim _{n \rightarrow \infty} \frac{n M_{n}}{b_{n}^{2}} \mathrm{E}\left(N_{c}\left(M_{n} w, M_{n}(w+t)\right]^{2} \mathbf{1}_{\left\{N_{0}\left(0, M_{n} w\right]<K \leq N_{0}\left(0, M_{n}(w+t)\right]\right\}} \mathbf{1}_{A_{n, M}}\right) \\
= & \frac{\alpha}{2-\alpha} \mathrm{E}\left(I(w+t)^{2-\alpha}-I(w)^{2-\alpha}\right)-2 \frac{\alpha}{\alpha-1} \mathrm{E}\left(I(w)\left(I(w)^{1-\alpha}-I(w+t)^{1-\alpha}\right)\right) \\
& +\mathrm{E}\left(I(w)^{2}\left(I(w)^{-\alpha}-I(w+t)^{-\alpha}\right)\right) .
\end{aligned}
$$

The integral over the complement of the event $A_{n, M}$ vanishes in the limit, as $M \rightarrow \infty$, in the same way as in Lemma 6.9.

The following theorem is needed to apply dominated convergence to establish the convergence of $\xi_{n}^{-}$in Lemma 5.2.

Theorem 6.1. Let Assumptions 2.1 and 2.2 hold. Then there exists a nonnegative measurable function $g: \mathbb{R}_{+} \rightarrow \mathbb{R}_{+}$such that $\int_{0}^{\infty} g(w) \mathrm{d} w<\infty$ and, for every $n \in \mathbb{N}$,

$$
\frac{n M_{n}}{b_{n}^{2}} \mathrm{E}\left(N_{c}\left(M_{n} w, M_{n}(w+1)\right]^{2}\right) \leq g(w) \text { for all } w>0 .
$$

Proof. The existence of the required function on the interval $(0, M]$ for an arbitrary $M>0$ follows from Lemma 6.14 , below, so we only need to construct a required function on the interval $(M, \infty)$. We define

$$
\begin{aligned}
& A_{n, w}=\left\{N_{0}\left(0, M_{n} w\right] \neq N_{0}\left(0, M_{n}(w+1)\right]\right\}, \\
& B_{n, w}=\left\{N_{0}\left(0, M_{n}\right]=N_{0}\left(0, M_{n} w\right]\right\} \cap A_{n, w}, \\
& C_{n, w}=\left\{N_{0}\left(0, M_{n}\right] \neq N_{0}\left(0, M_{n} w\right]\right\} \cap A_{n, w} .
\end{aligned}
$$

We have, for $w>M$,

$$
\begin{aligned}
\frac{n M_{n}}{b_{n}^{2}} \mathrm{E}\left(N_{c}\left(M_{n} w, M_{n}(w+1)\right]^{2}\right) \\
\leq \frac{n M_{n}}{b_{n}^{2}} \mathrm{E}\left(N_{c}\left(M_{n} w, M_{n}(w+1)\right]^{2} \mathbf{1}_{B_{n, w}}\right) \\
\quad+\frac{n M_{n}}{b_{n}^{2}} \mathrm{E}\left(N_{0}\left(M_{n} w, M_{n}(w+1)\right]^{2} \mathbf{1}_{\left\{K>N_{0}\left(0, M_{n} w\right]>0\right\}} \mathbf{1}_{C_{n, w}}\right) \\
=: J_{2,1}(n, w)+J_{2,2}(n, w) .
\end{aligned}
$$

Potter's inequality and Lemma 6.2 result in

$$
\begin{aligned}
& J_{2,2}(n, w) \\
& \quad \leq \mathrm{E}\left(\frac{N_{0}\left(M_{n} w, M_{n}(w+1)\right]^{2}}{\bar{F}\left(M_{n}\right)^{-2}}\left[C_{1}\left(\frac{N_{0}\left(0, M_{n} w\right]}{\bar{F}\left(M_{n}\right)^{-1}}\right)^{-\alpha_{1}}+C_{2}\left(\frac{N_{0}\left(0, M_{n} w\right]}{\bar{F}\left(M_{n}\right)^{-1}}\right)^{-\alpha_{2}}\right] \mathbf{1}_{C_{n, w}}\right) \\
& \quad \leq \mathrm{E}\left(\frac{\left(N_{0}\left(0, M_{n}\right]+1\right)^{2}}{\bar{F}\left(M_{n}\right)^{-2}}\right) \mathrm{E}\left(\left[C_{1}\left(\frac{N_{0}\left(0, M_{n} w\right]}{\bar{F}\left(M_{n}\right)^{-1}}\right)^{-\alpha_{1}}+C_{2}\left(\frac{N_{0}\left(0, M_{n} w\right]}{\bar{F}\left(M_{n}\right)^{-1}}\right)^{-\alpha_{2}}\right] \mathbf{1}_{C_{n, w}}\right) .
\end{aligned}
$$


By (6.3) we have, for large $n$,

$$
\begin{aligned}
\mathrm{E}\left(\frac{N_{0}\left(0, M_{n}\right]^{2}}{\bar{F}\left(M_{n}\right)^{-2}}\right) & =\frac{1}{\bar{F}\left(M_{n}\right)^{-2}} \int_{0}^{\infty} \mathrm{P}\left(N_{0}\left(0, M_{n}\right]^{2}>x\right) \mathrm{d} x \\
& \leq \frac{2}{\bar{F}\left(M_{n}\right)^{-2}} \int_{0}^{\infty} y \mathrm{P}\left(T_{\lfloor y\rfloor+1} \leq M_{n}\right) \mathrm{d} y \\
& \leq \frac{2}{\bar{F}\left(M_{n}\right)^{-2}} \int_{0}^{\infty} y \exp \left(-y \bar{F}\left(M_{n}\right)\right) \mathrm{d} y \\
& =2 \int_{0}^{\infty} z \mathrm{e}^{-z} \mathrm{~d} z \\
& <\infty
\end{aligned}
$$

Hence, (6.10), (6.11), and Proposition 6.1, below, show that $J_{2,2}(n, w)$ is uniformly in $n$ bounded from above by an integrable function on $[M, \infty)$. The fact that the same is true for $J_{2,1}(n, w)$ follows from Lemma 6.15, below.

The last piece needed to prove Lemma 5.2 is the next lemma.

Lemma 6.11. Let Assumptions 2.1 and 2.2 hold, and let $I_{3,1}(n)$ and $I_{3,2}(n)$ be as in (5.9). Then

(a) $\lim _{n \rightarrow \infty} I_{3,1}(n)=0$;

(b) $\lim _{n \rightarrow \infty} I_{3,2}(n)=0$.

Proof. (a) We assume, once again to ease the notation, that $\varepsilon=1$. Let $\theta>0$. We have

$$
\begin{aligned}
I_{3,1}(n) & \leq b_{n}^{-\theta} \frac{n M_{n}}{b_{n}^{2}} \int_{M}^{\infty} \mathrm{E}\left(N_{c}\left(M_{n} w, M_{n}(w+t)\right]^{2+\theta} \mathbf{1}_{\left\{N_{c}\left(M_{n} w, M_{n}(w+t)\right]>b_{n}\right\}}\right) \mathrm{d} w \\
& \leq b_{n}^{-\theta} \bar{F}\left(M_{n}\right)^{-\theta} \int_{M}^{\infty} \frac{1}{\mathrm{P}\left(K>\bar{F}\left(M_{n}\right)^{-1}\right)} \mathrm{E}\left(\left(\frac{N_{c}\left(M_{n} w, M_{n}(w+t)\right]}{\bar{F}\left(M_{n}\right)}\right)^{2+\theta}\right) \mathrm{d} w
\end{aligned}
$$

As in the proof of Theorem 6.1, the integral is bounded above by $C_{1} \int_{M}^{\infty} w^{-r} \mathrm{~d} w$ for some $C_{1}>0$ and $r>1$. Then by (6.1) we conclude that $I_{3,1}(n) \rightarrow 0$ as $n \rightarrow \infty$.

(b) For $I_{3,2}$, note that, by Lemma 6.3 ,

$$
\begin{aligned}
\mathrm{E}\left(\frac{N_{0}\left(0, M_{n}(M+t)\right]}{\bar{F}\left(M_{n}\right)^{-1}} \mathbf{1}_{\left\{N_{0}\left(0, M_{n}(M+t)\right]>b_{n}\right\}}\right) \\
=b_{n} \bar{F}\left(M_{n}\right) \mathrm{P}\left(N_{0}\left(0, M_{n}(M+t)\right]>b_{n}\right)+\bar{F}\left(M_{n}\right) \int_{b_{n}}^{\infty} \mathrm{P}\left(N_{0}\left(0, M_{n}(M+t)\right]>x\right) \mathrm{d} x \\
\leq b_{n} \bar{F}\left(M_{n}\right) \exp \left(-b_{n} \bar{F}\left(M_{n}(M+t)\right)\right)+\bar{F}\left(M_{n}\right) \int_{b_{n}}^{\infty} \exp \left(-x \bar{F}\left(M_{n}(M+t)\right)\right) \mathrm{d} x \\
=b_{n} \bar{F}\left(M_{n}\right) \exp \left(-b_{n} \bar{F}\left(M_{n}(M+t)\right)\right) \\
\quad+\bar{F}\left(M_{n}(M+t)\right)^{-1} \bar{F}\left(M_{n}\right) \exp \left(-b_{n} \bar{F}\left(M_{n}(M+t)\right)\right) .
\end{aligned}
$$


Therefore,

$$
\begin{aligned}
I_{3,2}(n) \leq & \frac{n M_{n}}{b_{n}} M \mathrm{E}\left(N_{0}\left(0, M_{n}(M+t)\right] \mathbf{1}_{\left\{N_{0}\left(0, M_{n}(M+t)\right]>b_{n}\right\}} \mathbf{1}_{\left\{K>b_{n}\right\}}\right) \\
\leq & M \bar{F}\left(M_{n}\right) b_{n} \mathrm{E}\left(\frac{N_{0}\left(0, M_{n}(M+t)\right]}{\bar{F}\left(M_{n}\right)^{-1}} \mathbf{1}_{\left\{N_{0}\left(0, M_{n}(M+t)\right]>b_{n}\right\}}\right) \frac{\mathrm{P}\left(K>b_{n}\right)}{\mathrm{P}\left(K>\bar{F}\left(M_{n}\right)^{-1}\right)} \\
\leq & C_{1}\left[\left(\bar{F}\left(M_{n}\right) b_{n}\right)^{2}+\bar{F}\left(M_{n}(M+t)\right)^{-1} \bar{F}\left(M_{n}\right) \bar{F}\left(M_{n}\right) b_{n}\right] \\
& \times \exp \left(-b_{n} \bar{F}\left(M_{n}(M+t)\right)\right)\left(b_{n} \bar{F}\left(M_{n}\right)\right)^{-\alpha_{1}} \\
\rightarrow & 0 \quad \text { as } n \rightarrow \infty,
\end{aligned}
$$

by (6.1).

\subsection{Auxiliary results for the proof of Theorem 6.1}

The following proposition is the first ingredient in the proof of Theorem 6.1.

Proposition 6.1. Let $\eta>1$ and $M>1$, and suppose that Assumptions 2.1 and 2.2 hold. Then there exists a nonnegative measurable function $g: \mathbb{R}_{+} \rightarrow \mathbb{R}_{+}$such that $\int_{M}^{\infty} g(w) \mathrm{d} w<\infty$ and, for every $n \in \mathbb{N}$,

$$
\mathrm{E}\left(\left(\frac{N_{0}\left(0, M_{n} w\right]}{\bar{F}\left(M_{n}\right)^{-1}}\right)^{-\eta} \mathbf{1}_{\left\{N_{0}\left(0, M_{n}\right] \neq N_{0}\left(0, M_{n} w\right] \neq N_{0}\left(0, M_{n}(w+1)\right]\right\}}\right) \leq g(w) \text { for all } w \geq M .
$$

The statement follows from Lemma 6.12 and Lemma 6.13, below.

Lemma 6.12. Let $\eta>1$ and $M>1$, and suppose that Assumptions 2.1 and 2.2 hold. Then there exists a nonnegative measurable function $g: \mathbb{R}_{+} \rightarrow \mathbb{R}_{+}$such that $\int_{M}^{\infty} g(w) \mathrm{d} w<\infty$ and, for every $n \in \mathbb{N}$,

$$
\mathrm{E}\left(\left(\frac{N_{0}\left(0, M_{n} w\right]}{\bar{F}\left(M_{n}\right)^{-1}}\right)^{-\eta} \mathbf{1}_{\left\{N_{0}\left(0, M_{n}(w-1)\right] \neq N_{0}\left(0, M_{n} w\right]\right\}}\right) \leq g(w) \text { for all } w \geq M .
$$

Proof. Let $w \geq M$ and $n$ be large enough that $M_{n}^{-1} \leq 2^{-1}$. We have

$$
\begin{aligned}
J_{1}(n, w) & :=\mathrm{E}\left(\left(\frac{N_{0}\left(0, M_{n} w\right]}{\bar{F}\left(M_{n}\right)^{-1}}\right)^{-\eta} \mathbf{1}_{\left\{N_{0}\left(0, M_{n}(w-1)\right] \neq N_{0}\left(0, M_{n} w\right]\right\}}\right) \\
& =\int_{M_{n}(w-1)}^{M_{n} w} \bar{F}\left(M_{n} w-y\right) \sum_{j=1}^{\infty}\left(\frac{j}{\bar{F}\left(M_{n}\right)^{-1}}\right)^{-\eta} \mathrm{P}\left(T_{j} \in \mathrm{d} y\right) \\
& =\left[\int_{M_{n}(w-1)}^{M_{n} w-2}+\int_{M_{n} w-2}^{M_{n} w}\right] \bar{F}\left(M_{n} w-y\right) \sum_{j=1}^{\infty}\left(\frac{j}{\bar{F}\left(M_{n}\right)^{-1}}\right)^{-\eta} \mathrm{P}\left(T_{j} \in \mathrm{d} y\right) \\
& =: J_{1,1}(n, \omega)+J_{1,2}(n, \omega) .
\end{aligned}
$$

Now,

$$
\begin{aligned}
J_{1,1}(n, \omega) \leq & \sum_{k=\left\lfloor M_{n}(w-1)\right\rfloor-1}^{\left\lceil M_{n} w-2\right\rceil-1} \bar{F}\left(M_{n}\left(w-\frac{k+1}{M_{n}}\right)\right) \\
& \times \sum_{j=1}^{\infty}\left(\frac{j}{\bar{F}\left(M_{n}\right)^{-1}}\right)^{-\eta}\left[\mathrm{P}\left(T_{j} \leq k+1\right)-\mathrm{P}\left(T_{j} \leq k\right)\right] .
\end{aligned}
$$


Since $\bar{F}$ is regularly varying with index $-1 / \beta$, by Potter's inequality, there exists a constant $0 \leq C_{1}<\infty$ such that

$$
\begin{aligned}
J_{1,1}(n, \omega) \leq & C_{1} \sum_{k=\left\lfloor M_{n}(w-1)\right\rfloor}^{\left\lceil M_{n} w-2\right\rceil-1}\left(w-\frac{k+1}{M_{n}}\right)^{-1 / \beta_{1}} \frac{\bar{F}\left(M_{n}\right)^{1-\eta}}{k \bar{F}(k)^{1-\eta}} k \bar{F}(k)^{1-\eta} \\
& \times \sum_{j=1}^{\infty} j^{-\eta}\left[\mathrm{P}\left(T_{j} \leq k+1\right)-\mathrm{P}\left(T_{j} \leq k\right)\right] .
\end{aligned}
$$

Using Lemma 4.1, we obtain

$$
J_{1,1}(n, \omega) \leq C_{2} \sum_{k=\left\lfloor M_{n}(w-1)\right\rfloor}^{\left\lceil M_{n} w-2\right\rceil-1}\left(w-\frac{k+1}{M_{n}}\right)^{-1 / \beta_{1}} \frac{\bar{F}\left(M_{n}\right)^{1-\eta}}{k \bar{F}(k)^{1-\eta}} .
$$

Again, taking the regular variation of $\bar{F}$ and Potter's theorem into account yields

$$
\begin{aligned}
J_{1,1}(n, \omega) & \leq C_{3} \sum_{k=\left\lfloor M_{n}(w-1)\right\rfloor}^{\left\lceil M_{n} w-2\right\rceil-1}\left(w-\frac{k+1}{M_{n}}\right)^{-1 / \beta_{1}} \frac{1}{k}\left(\frac{k}{M_{n}}\right)^{(1-\eta) / \beta_{1}} \\
& =C_{3} \sum_{k=\left\lfloor M_{n}(w-1)\right\rfloor}^{\left\lceil M_{n} w-2\right\rceil-1} \frac{1}{M_{n}}\left(w-\frac{k+1}{M_{n}}\right)^{-1 / \beta_{1}}\left(\frac{k}{M_{n}}\right)^{(1-\eta) / \beta_{1}-1} \\
& \leq C_{4} \int_{w-1}^{w}(w-z)^{-1 / \beta_{1}} z^{(1-\eta) / \beta_{1}-1} \mathrm{~d} z \\
& \leq C_{5} w^{(1-\eta) / \beta_{1}-1}
\end{aligned}
$$

which is an integrable function on $[M, \infty)$ since $\eta>1$.

Finally, using, once again, Lemma 4.1, we obtain

$$
\begin{aligned}
J_{1,2}(n, \omega) & \leq \bar{F}\left(M_{n}\right)^{-\eta} \sum_{j=1}^{\infty} j^{-\eta}\left[\mathrm{P}\left(T_{j} \leq M_{n} w\right)-\mathrm{P}\left(T_{j} \leq M_{n} w-2\right)\right] \\
& \leq C_{6} \bar{F}\left(M_{n}\right)^{-\eta} \frac{1}{M_{n} w} \bar{F}\left(M_{n} w\right)^{\eta-1} \\
& \leq C_{7} \frac{1}{M_{n} \bar{F}\left(M_{n}\right)} w^{(1-\eta) / \beta_{1}-1}
\end{aligned}
$$

which is uniformly bounded by an integrable function.

Lemma 6.13. Let $\eta>1$ and $M>1$, and suppose that Assumptions 2.1 and 2.2 hold. Then there exists a nonnegative measurable function $g: \mathbb{R}_{+} \rightarrow \mathbb{R}_{+}$such that $\int_{M}^{\infty} g(w) \mathrm{d} w<\infty$ and, for every $n \in \mathbb{N}$,

$$
\begin{aligned}
& \mathrm{E}\left(\left(\frac{N_{0}\left(0, M_{n} w\right]}{\bar{F}\left(M_{n}\right)^{-1}}\right)^{-\eta} \mathbf{1}_{\left\{N_{0}\left(0, M_{n}\right] \neq N_{0}\left(0, M_{n}(w-1)\right]=N_{0}\left(0, M_{n} w\right] \neq N_{0}\left(0, M_{n}(w+1)\right]\right\}}\right) \\
& \quad \leq g(w) \text { for all } w \geq M .
\end{aligned}
$$


Proof. As in the previous lemma,

$$
\begin{aligned}
J_{1}(n, w):= & \mathrm{E}\left(\left(\frac{N_{0}\left(0, M_{n} w\right]}{\bar{F}\left(M_{n}\right)^{-1}}\right)^{-\eta} \mathbf{1}_{\left\{N_{0}\left(0, M_{n}\right] \neq N_{0}\left(0, M_{n}(w-1)\right]=N_{0}\left(0, M_{n} w\right] \neq N_{0}\left(0, M_{n}(w+1)\right]\right\}}\right) \\
= & \int_{M_{n}}^{M_{n}(w-1)}\left[\bar{F}\left(M_{n} w-y\right)-\bar{F}\left(M_{n}(w+1)-y\right)\right] \\
& \times \sum_{j=1}^{\infty}\left(\frac{j}{\bar{F}\left(M_{n}\right)^{-1}}\right)^{-\eta} \mathrm{P}\left(T_{j} \in \mathrm{d} y\right) \\
\leq & \sum_{k=\left\lfloor M_{n}\right\rfloor}^{\left\lceil M_{n}(w-1)\right\rceil-1}\left[\bar{F}\left(M_{n} w-k-1\right)-\bar{F}\left(M_{n} w+M_{n}-k\right)\right] \\
& \times \sum_{j=1}^{\infty}\left(\frac{j}{\bar{F}\left(M_{n}\right)^{-1}}\right)^{-\eta}\left[\mathrm{P}\left(T_{j} \leq k+1\right)-\mathrm{P}\left(T_{j} \leq k\right)\right] .
\end{aligned}
$$

By Lemma 4.1 we have, for large $n$,

$$
J_{1}(n, w) \leq C_{1} \sum_{k=\left\lfloor M_{n}\right\rfloor}^{\left\lceil M_{n}(w-1)\right\rceil-1} \frac{\bar{F}\left(M_{n} w-k-1\right)-\bar{F}\left(M_{n} w+M_{n}-k\right)}{\bar{F}\left(M_{n}\right)} \frac{\bar{F}\left(M_{n}\right)^{1-\eta}}{k \bar{F}(k)^{1-\eta}} .
$$

Note that, for every $k$ in the above sum, by Assumption 2.2,

$$
\begin{aligned}
& \bar{F}\left(M_{n} w-k-1\right)-\bar{F}\left(M_{n} w+M_{n}-k\right) \\
& \leq \sum_{j=-1}^{\left\lceil M_{n}\right\rceil-1}\left[\bar{F}\left(M_{n} w-k+j\right)-\bar{F}\left(M_{n} w-k+j+1\right)\right] \\
& \leq C_{2} \sum_{j=-1}^{\left\lceil M_{n}\right\rceil-1} \frac{\bar{F}\left(M_{n} w-k+j\right)}{M_{n} w-k+j} \\
& \leq C_{3} M_{n} \frac{\bar{F}\left(M_{n} w-k-1\right)}{M_{n} w-k-1} .
\end{aligned}
$$

We conclude by Potter's theorem that, for large $n$ and every $k$ as above,

$$
\frac{\bar{F}\left(M_{n} w-k-1\right)-\bar{F}\left(M_{n} w+M_{n}-k\right)}{\bar{F}\left(M_{n}\right)} \leq C_{4}\left(w-\frac{k+1}{M_{n}}\right)^{-1 / \beta_{2}-1} .
$$

Hence, we obtain

$$
J_{1}(n, w) \leq C_{4} \sum_{k=\left\lfloor M_{n}\right\rfloor}^{\left\lceil M_{n}(w-1)\right\rceil-1}\left(w-\frac{k+1}{M_{n}}\right)^{-1 / \beta_{2}-1} \frac{\bar{F}\left(M_{n}\right)^{1-\eta}}{k \bar{F}(k)^{1-\eta}} .
$$

Similar calculations as in (6.12) result in

$$
J_{1}(n, w) \leq C_{5} \int_{1}^{w-1}(w-z)^{-1 / \beta_{2}-1} z^{(1-\eta) / \beta_{2}-1} \mathrm{~d} z \leq C_{6} w^{-(\eta-1) / \beta_{2}-1},
$$

as an easy computation shows. This is an integrable function on $[M, \infty)$. 
The final two lemmas needed for the proof of Theorem 6.1 follow.

Lemma 6.14. Let $M>0$, and suppose that Assumption 2.1 holds. Then there exists a positive constant $C<\infty$ such that, for every $n \in \mathbb{N}$,

$$
\frac{n M_{n}}{b_{n}^{2}} \mathrm{E}\left(N_{c}\left(M_{n} w, M_{n}(w+1)\right]^{2}\right) \leq C \quad \text { for all } w \leq M
$$

Proof. It is clearly enough to establish the required bound for large enough $n$. By Potter's inequality and Karamata's theorem, we obtain, for all large enough $n$ and $0<w \leq M$,

$$
\begin{aligned}
& \frac{n M_{n}}{b_{n}^{2}} \mathrm{E}\left(N_{c}\left(M_{n} w, M_{n}(w+1)\right]^{2}\right) \\
& \leq \frac{n M_{n}}{b_{n}^{2}} \mathrm{E}\left(N_{c}\left(0, M_{n}(M+1)\right]^{2}\right) \\
&=\frac{n M_{n}}{b_{n}^{2}} \mathrm{E}\left(N_{0}\left(0, M_{n}(M+1)\right]^{2} \mathbf{1}_{\left.\left\{K>N_{0}\left(0, M_{n}(M+1)\right]>0\right\}\right)}\right. \\
& \quad+\frac{n M_{n}}{b_{n}^{2}} \mathrm{E}\left(K^{2} \mathbf{1}_{\left\{K \leq N_{0}\left(0, M_{n}(M+1)\right]\right\}}\right) \\
& \leq C_{1} \mathrm{E}\left(\left(\frac{N_{0}\left(0, M_{n}(M+1)\right]}{\bar{F}\left(M_{n}\right)^{-1}}\right)^{2-\alpha_{1}}\right)+C_{2} \mathrm{E}\left(\left(\frac{N_{0}\left(0, M_{n}(M+1)\right]}{\bar{F}\left(M_{n}\right)^{-1}}\right)^{2-\alpha_{2}}\right) .
\end{aligned}
$$

The right-hand side is bounded for large enough $n$ by computations similar to (6.11).

Lemma 6.15. Let $M>1$, and suppose that Assumptions 2.1 and 2.2 hold. Then there exists a nonnegative measurable function $g: \mathbb{R}_{+} \rightarrow \mathbb{R}_{+}$such that $\int_{M}^{\infty} g(w) \mathrm{d} w<\infty$ and, for every $n \in \mathbb{N}$,

$$
\frac{n M_{n}}{b_{n}^{2}} \mathrm{E}\left(N_{c}\left(M_{n} w, M_{n}(w+1)\right]^{2} \mathbf{1}_{\left\{N_{0}\left(0, M_{n}\right]=N_{0}\left(0, M_{n} w\right] \neq N_{0}\left(0, M_{n}(w+1)\right]\right\}}\right) \leq g(w)
$$

for all $w \geq M$.

Proof. We define $B_{n, w}:=\left\{N_{0}\left(0, M_{n}\right]=N_{0}\left(0, M_{n} w\right] \neq N_{0}\left(0, M_{n}(w+1)\right]\right\}$. Note that, by Lemma 6.2,

$$
\frac{n M_{n}}{b_{n}^{2}} \mathrm{E}\left(N_{c}\left(M_{n} w, M_{n}(w+1)\right]^{2} \mathbf{1}_{B_{n, w}}\right) \leq \frac{n M_{n}}{b_{n}^{2}} \mathrm{E}\left(\min \left(N_{0}\left(0, M_{n}\right], K\right)^{2}\right) \mathrm{P}\left(B_{n, w}\right) .
$$

Furthermore,

$$
\begin{aligned}
\mathrm{E}\left(\min \left(N_{0}\left(0, M_{n}\right], K\right)^{2}\right) & =2 \int_{0}^{\infty} t \mathrm{P}\left(N_{0}\left(0, M_{n}\right]>t\right) \mathrm{P}(K>t) \mathrm{d} t \\
& =2\left[\int_{0}^{\bar{F}\left(M_{n}\right)^{-1}}+\int_{\bar{F}\left(M_{n}\right)^{-1}}^{\infty}\right] t \mathrm{P}\left(N_{0}\left(0, M_{n}\right]>t\right) \mathrm{P}(K>t) \mathrm{d} t .
\end{aligned}
$$

Since, by Karamata's theorem, as $n \rightarrow \infty$,

$$
\begin{aligned}
\int_{0}^{\bar{F}\left(M_{n}\right)^{-1}} t \mathrm{P}\left(N_{0}\left(0, M_{n}\right]>t\right) \mathrm{P}(K>t) \mathrm{d} t & \leq \int_{0}^{\bar{F}\left(M_{n}\right)^{-1}} t \mathrm{P}(K>t) \mathrm{d} t \\
& \sim C_{1}\left(\bar{F}\left(M_{n}\right)^{-1}\right)^{2} \mathrm{P}\left(K>\bar{F}\left(M_{n}\right)^{-1}\right)
\end{aligned}
$$


and, by (6.11),

$$
\begin{aligned}
\int_{\bar{F}\left(M_{n}\right)^{-1}}^{\infty} t \mathrm{P}\left(N_{0}\left(0, M_{n}\right]>t\right) \mathrm{P}(K>t) \mathrm{d} t & \leq C_{2} \mathrm{P}\left(K>\bar{F}\left(M_{n}\right)^{-1}\right) \mathrm{E}\left(N_{0}\left(0, M_{n}\right]^{2}\right) \\
& \leq C_{3}\left(\bar{F}\left(M_{n}\right)^{-1}\right)^{2} \mathrm{P}\left(K>\bar{F}\left(M_{n}\right)^{-1}\right),
\end{aligned}
$$

we have the bound

$$
\mathrm{E}\left(\min \left(N_{0}\left(0, M_{n}\right], K\right)^{2}\right) \leq C_{4}\left(\bar{F}\left(M_{n}\right)^{-1}\right)^{2} \mathrm{P}\left(K>\bar{F}\left(M_{n}\right)^{-1}\right) .
$$

On the other hand, by Assumption 2.2 and the same arguments as in (6.11),

$$
\begin{aligned}
\mathrm{P}\left(B_{n, w}\right) & =\mathrm{P}\left(N_{0}\left(0, M_{n}\right]=N_{0}\left(0, M_{n} w\right] \neq N_{0}\left(0, M_{n}(w+1)\right]\right) \\
& =\sum_{j=0}^{\infty} \int_{0}^{M_{n}}\left[\bar{F}\left(M_{n} w-y\right)-\bar{F}\left(M_{n} w+M_{n}-y\right)\right] \mathrm{P}\left(T_{j} \in \mathrm{d} y\right) \\
& \leq\left[\bar{F}\left(M_{n} w-M_{n}\right)-\bar{F}\left(M_{n} w+M_{n}\right)\right] \mathrm{E}\left(N_{0}\left(0, M_{n}\right]\right) \\
& \leq \sum_{k=0}^{\left\lfloor M_{n}\right\rfloor}\left[\bar{F}\left(M_{n} w-M_{n}+k\right)-\bar{F}\left(M_{n} w+k+1\right)\right]\left[C_{5} \bar{F}\left(M_{n}\right)^{-1}\right] \\
& \leq C_{6} \bar{F}\left(M_{n}\right)^{-1} \sum_{k=0}^{\left\lfloor M_{n}\right\rfloor} \frac{\bar{F}\left(M_{n} w-M_{n}+k\right)}{M_{n} w-M_{n}+k} \\
& \leq C_{7} \bar{F}\left(M_{n}\right)^{-1} M_{n} \frac{\bar{F}\left(M_{n} w-M_{n}\right)}{M_{n} w-M_{n}} \\
& \leq C_{8} w^{-1} \frac{\bar{F}\left(M_{n} w\right)}{\bar{F}\left(M_{n}\right)} .
\end{aligned}
$$

We conclude that

$$
\frac{n M_{n}}{b_{n}^{2}} \mathrm{E}\left(N_{c}\left(M_{n} w, M_{n}(w+1)\right]^{2} \mathbf{1}_{B_{n, w}}\right) \leq C_{9} w^{-1} \frac{\bar{F}\left(M_{n} w\right)}{\bar{F}\left(M_{n}\right)} \leq C_{10} w^{-1-1 / \beta_{2}},
$$

which is an integrable function on $[M, \infty)$.

\section{Acknowledgements}

Parts of the paper were written while the first author was visiting the Department of Operations Research and Information Engineering at Cornell University. She takes pleasure in thanking her colleagues there for their pleasant hospitality. Financial support from the Deutsche Forschungsgemeinschaft through a research grant is gratefully acknowledged. Samorodnitsky's research was partially supported by an NSA grant MSPF-05G-049 and an ARO grant W911NF07-1-0078 at Cornell University.

\section{References}

Anderson, K. K. And Athreya, K. B. (1988). A strong renewal theorem for generalized renewal functions in the infinite mean case. Prob. Theory Relat. Fields 77, 471-479.

Bingham, N. H., Goldie, C. M. And Teugels, J. L. (1987). Regular Variation (Encyclopaedia Math. Appl. 27).

Cambridge University Press. 
D’Auria, B. ANd Samorodnitsky, G. (2005). Limit behavior of fluid queues and networks. Operat. Res. 53, $933-945$.

Delgado, R. (2007). A reflected fBm limit for fluid models with ON/OFF sources under heavy traffic. Stoch. Process. Appl. 117, 188-201.

Denisov, D., Dieker, A. B. And Shneer, V. (2008). Large deviations for random walks under subexponentiality: the big-jump domain. Ann. Prob. 36, 1946-1991.

Doney, R. A. (1997). One-sided local large deviation and renewal theorems in the case of infinite mean. Prob. Theory Relat. Fields 107, 451-465.

Fä̈, G., González-Arévalo, B., Mikosch, T. and Samorodnitsky, G. (2006). Modeling teletraffic arrivals by a Poisson cluster process. Queueing Systems 54, 121-140.

Gaigalas, R. and KaJ, I. (2003). Convergence of scaled renewal processes and a packet arrival model. Bernoulli 9 , 671-703.

KAJ, I., LeSKelä, L., Norros, I. AND SCHMIDT, V. (2007). Scaling limits for random fields with long-range dependence. Ann. Prob. 35, 528-550.

Kallenberg, O. (2002). Foundations of Modern Probability, 2nd edn. Springer, New York.

Meerschaert, M. M. AND Scheffler, H.-P. (2004). Limit theorems for continuous-time random walks with infinite mean waiting times. J. Appl. Prob. 41, 623-638.

Mikosch, T. and Samorodnitsky, G. (2007). Scaling limits for workload process. Operat. Res. 32, 890-918.

Mikosch, T., Resnick, S., Rootzén, H. and Stegeman, A. (2002). Is network traffic approximated by stable Lévy motion or fractional Brownian motion? Ann. Appl. Prob. 12, 23-68.

Park, K. AND Willinger, W. (2000). Self-Similar Network Traffic and Performance Evaluation. John Wiley, New York.

Petrov, V. V. (1975). Sums of Independent Random Variables. Springer, New York.

Resnick, S. I. (2007). Heavy-Tail Phenomena. Springer, New York. 